(Aus dem pharmakologischen Laboratorium

von Professor Joh. Dogiel an der Universität zu Kasan.)

\title{
Beiträge \\ zur Erforschung des Sympathicuseinflusses auf die contralaterale Pupille.
}

Von

\section{Dr. T. Tümianzew.}

(Mit einer Tafel.)

Die vorliegende, auf Vorsehlag und unter Leitung von Prof. Joh. Dogiel ausgeführte Arbeit zerfällt naturgemäss in zwei Abschnitte - einen anatomischen und einen physiologischen. Im ersteren will ich an der Hand der in der Literatur niedergelegten Daten und eigener Untersuchungen den gegenwärtigen Zustand unserer Kenntnisse über die Bahnen der Seh- und Pupillenfasern darstellen, im zweiten aber den Einfluss, welchen der N. sympathicus auf die contralaterale Pupille ausübt, experimentell demonstriren.

I. Anatomisches. Die Anwendung des Methylenblau bei Katzen und Hunden und der Marchi'schen Methode bei Kaninchen hat mich überzeugt, dass im Chiasma dieser Thiere nur eine partielle Kreuzung der Opticusfasern stattfindet. Wie verhalten sich aber die die Pupillenbewegung vermittelnden Nervenfasern in Chiasma? Gudden ${ }^{1}$ ) war der Erste, welcher im Sehnerven und in dem Tractus opt. zwei morphologisch und functionell verschiedene Faserarten, Seh- und Pupillarfasern, unterschied. Er fand, dass bei Kaninchen nach Läsion des lateralen Kniehöckers die Pupille des entgegengesetzten, blinden Auges nicht mehr auf Licht reagirt. Nach der Zerstörung der oberen Vierhügel beobachtete er bei Kaninchen Blindheit auf beiden Augen, während die Pupillenreaction normal blieb. Daraus folgt, dass die vorderen Erhebungen des Vierhügels ausschliesslich Sehcentra, das Corpus genicul. lat. aber das Centrum für die Pupillenfasern darstellt. Auch nach den Zerstörungen des hinteren Sehhügelabschnittes beobachtete man Pupillenstörungen. Die 
Pupillenfasern zeichnen sich nach Gudden ${ }^{2}$ ) durch ihre Dicke aus. Entfernte er bei einem neugeborenen Kaninchen die oberen Vierhügel, so erwies es sich, dass das contralaterale Auge des erwachsenen Thieres blind, die Pupillenreaction aber erhalten war. Die im Opticus des blinden Auges vorgefundenen dicken Fasern nannte $\mathrm{Gu}$ d d en Pupillarfasern, im Gegensatz zu den mehr dünneren, im oberen Vierhügel endigenden Sehfasern. Aehnliche Verhältnisse hat er auch bei Katzen constatirt ${ }^{1}$ ). Die Abstammung der dünnen Sehfasern aus dem vorderen Vierhügel hat $\mathrm{M} \mathrm{Onakow}^{3}$ ) bestätigt: Es fragt sich nun, ob diese Pupillenfasern sich im Chiasma kreuzen? W1. Bechterew war Anfangs der Meinung, dass die Pupillenfasern keiner Kreuzung unterliegen ${ }^{4}$ ); hernach hat er aber eine solche Kreuzung im Chiasma wieder angenommen ${ }^{5}$ ). Ist aber nun die im Chiasma stattfindende Kreuzung der Pupillenfasern eine totale oder eine partielle? Soweit mir bekannt, wird für Katze und Hund allgemein eine totale Kreuzung angenommen. Nicati ${ }^{6}$ ) hat von der Maulhöhle aus mit einem gebogenen Bistouri das Chiasma in der Mittellinie von vorn nach hinten durchschnitten. Nach seinem zweiten, einzig gelungenen Versuch blieb die Lichtreaction der Pupillen bestehen. Bechterew ${ }^{7}$ ) hat dieselbe Operation an Hunden ausgeführt und sah, dass die Pupillenweite normal und die Lichtreaction derselben erhalten blieb. Hernach ${ }^{8}$ ) finden wir bei ihm die Angabe, dass beim Hunde nach der Durehschneidung des einen Tractus opt. beide Pupillen wohl zweifellos gut auf das Licht reagiren, dennoch aber am contralateralen Auge die Pupille weniger weit als am entsprechenden Auge erscheint, und die hemiopische Lichtreaction zur Beobachtung gelangt. Sinani ${ }^{9}$ ) durchschnitt beim Hunde den Tractus opt. und beobachtete, dass die Pupillen gleich weit blieben und bei der Annäherung des Lichtes sich gleichmässig verengten. Wurde aber jedes Auge für sich auf den Lichtreiz untersucht, wobei das andere Auge verdeckt war, so bemerkte er, dass die Pupille an der operirten Seite sich stärker zu verengern sehien, als an der anderen. Auf Grund seiner Durchschneidungsversuche des einen Tractus opt. der Katze glaubt $\mathrm{Gudde} \mathrm{n}^{2}$ ), dass die dicken Opticusfasern (Pupillarfasern) einer partiellen Kreuzung unterliegen. Pribytkow ${ }^{10}$ ) hat den Tractus opt. bei Hunden und Katzen durchschnitten und keine Veränderung in der Weite und in der Reaction der Pupillen erhalten. In Bezug auf Hunde und Katzen wären somit die Untersucher im Ganzen damit einverstanden, dass die Pupillenfasern im 
Chiasma nur einer partiellen Kreuzung unterliegen. Nicht so ist das der Fall mit den Literaturangaben über das Verhalten der Pupillenfasern im Chiasma des Kaninchens.

Longet ${ }^{11}$ ) reizte beim Kaninchen den centralen Opticusstumpf und erhielt meist Verengerung beider Pupillen. Die in der Orbita vorgenommene Opticusreizung gab $\mathrm{Budg \textrm {e } ^ { 1 2 }}$ ) eine Verengerung der Pupille an der entsprechenden Seite. $\mathrm{Knoll}^{13}$ ) fand nach der Durchschneidung des einen Tractus opt. Erweiterung und vollkommene Reactionslosigkeit nur an der contralateralen Pupille, woraus er auf eine totale Kreuzung im Chiasma der Kaninchen schloss. Eckhardt ${ }^{14}$ ) ist derselben Meinung. Darkschewitsch ${ }^{15}$ ) zieht aus seinen Versuchen mit Verletzung des Sehhügels und der Seitenwand des dritten Ventrikels und aus seinen Beobachtungen an den nach der Sinani'schen Methode operirten Kaninchen den Schluss, dass ein Theil der Pupillenfasern ungekreuzt bleibe und im Tractus opt. derselben Seite verlaufe. Gudden ${ }^{1}$ ) fand, dass nach der Verletzung des äusseren Kniehöckers bei Kaninchen nur die Pupille des contralateralen Auges nicht mehr auf das Licht reagirt. Ausgehend von den Knoll'sehen und Gudden'schen Versuchen nimmt Stei$\mathrm{nach}{ }^{16}$ ) an, dass das ungekreuzte Bündel im Chiasma des Kaninchens keine Pupillenfasern enthalte. Er schreibt: „Wenn das ungekreuzte Bündel Pupillarfasern enthielte - ich will diesen nunmehr gegenstandslos gewordenen Einwand nur per parenthesin einflechten -, so hätte in allen jenen Fällen, wo in Folge von gelungenen Operationsacten beim Kaninchen ein Auge bloss durch den ungekreuzten Opticusantheil mit dem primären Centrum in Verbindung, stand, die Lichtreizung dieses Auges unter der nothwendigen Voraussetzung der totalen Pupillarfaserkreuzung im Oculomotorius durchaus nicht Reaction seiner Pupille, sondern gerade der entgegengesetzten, also nur eine consensuelle Reaction auslösen müssen, was natürlich nie, ebensowenig wie bei den normalen Thieren eingetreten ist " (S. 333). Entsprechende eigene Versuche werden jedoch nicht angeführt. Somit glauben einige Autoren, dass die Kreuzung der Pupillenfasern im Chiasma des Kaninchens eine totale, andere aber, dass sie eine partielle ist.

In Bezug auf den Verlauf der Pupillenfasern im Tractus opticus ist Wl. Bechtere $\mathrm{w}^{4}$ ) von seiner früheren Meinung, dass dieselben nicht im Tract. opt. vorhanden seien, sondern hinter dem Chiasma in die graue Bekleidungsschicht des dritten Ventrikels dringen und 
zum Oculomotoriuskern gehen, zurïckgekommen und nimmt gegenwärtig an, dass sie im Tract. opt. eine Strecke zurücklegen ${ }^{5}$ ). Auch Henschen ${ }^{17}$ ) behauptet auf Grund einer Reihe von pathologischanatomischen und klinischen Beobachtungen, dass die Pupillenfasern im dorso-lateralen Theil des Tract. opt. verlaufen.

Grösser ist der Widerspruch über den weiteren Verlauf der Pupillenfasern. Nach Hertwig ${ }^{13}$ ) lähmt die einseitige starke Verletzung und die totale Exstirpation des Vierhügels die Pupille an der anderen Seite. Auch Flourens ${ }^{18}$ ) nimmt auf Grund seiner Versuche an, dass die Pupillenreactionen nach genügender Verletzung des Vierhügels aufhören, zu welchem Schluss auch Longet ${ }^{11}$ ) gelangt ist. $\mathrm{Budg} \mathrm{e}{ }^{{ }^{2}}$ ) schreibt wohl dem Vierhügel einen bestimmten Einfluss auf die Pupillen zu, möchte aber auf Grund seiner Versuche nicht entscheiden, ob der Opticus oder der Oculomotorius oder beide zusammen aus demselben entspringen. Renzi ${ }^{15}$ ) aber hat nach Verletzung und Exstirpation des Vierhügels, wenn zufällige Läsionen bei der Operation vermieden worden waren, niemals die Pupillenreaction aufhören sehen. Nach der Operation gelangte sogar eine geringe Verengerung der entgegengesetzten Pupille zur Beobachtung. $\mathrm{Knol1}{ }^{13}$ ) fand, dass die durch den Vierhügel angelegten Schnitte, falls die Sehnerven dabei geschont wurden, keine Wirkung auf die reflectorischen Pupillenbewegungen hatten. Meynert ${ }^{19}$ ) glaubt, dass die Reizungsübermittelung vom Opticus auf den Oculomotorius durch die dünnen radialen Fasern im vorderen Vierhügelgebiet geschieht. Hiergegen sprechen jedoch die oben angeführten Beobachtungen von Gudden, Bechterew and Darkschewitsch. Letzterer hat sich überzeugen können ${ }^{15}$ ), dass die Verletzung des vorderen Vierhügels, falls die Commissura cerebri post. unversehrt blieb, gar keine Veränderungen der normalen Pupillenbewegungen auf Lichtreiz zur Folge hat. Weiter unten gehen die radialen Fasern in die fontänenförmige Kreuzung Meynert's über ${ }^{5}$ ); ein Theil der Fasern dieses Systems sendet in dorsaler Richtung Seitenzweige zu den Oculomotoriuskernen. Die Fasern der fontänenförmigen Kreuzung erscheinen jedoch auch beim Maulwurf gut ausgebildet, bei welchem die Sehnerven und die Tract. opt. doch nur rudimentär sind. Auch nach Henschen ${ }^{17}$ ) gehören die radialen Fasern Meynert's nicht der Pupille an, da sie bei der Atrophie des Tract. opt. nicht mitatrophiren. Obersteiner ${ }^{20}$ ) aber und Koelliker ${ }^{21}$ ) theilen die Meynert'sche Ansicht. 
Beiträge zur Erforschung des Sympathicuseinflusses auf die contral. Pupille. 203

Hensen und Völkers ${ }^{22}$ ) erhielten beim Hunde bei der Reizung des Bodens vom dritten Ventrikel Pupillenverengerung an der entsprechenden Seite. B ec b t e r e w ${ }^{23}$ ) konnte aber bei seinen Reizungen und Zerstörungen des Vierhügels bei verschiedenen Thieren sich davon überzeugen, dass eine möglichst isolirte Zerstörung des Vierhügels nicht von starken Veränderungen in der Pupillenweite begleitet ist. Stilling ${ }^{24}$ ) studirte den Verlauf der Primärbündel des Sehnerven, indem er normale, macerirte Präparate zerzupfte. Er beschreibt eine besondere absteigende Opticuswurzel, welche aus dem Tract. opt. in den Grosshirnschenkel, sogleich hinter dem lateralen Kniehöcker, tritt, oder in dem Raum zwischen dem Grosshirnschenkel und dem Corpus geniculatum laterale verläuft. Einen zum Oculomotoriuskern tretenden Theil dieser Fasern hält Stilling für Pupillenfasern. Zum Oculomotoriuskern geht wahrscheinlich auch der aus dem oberen Vierhügel entspringende Tractus peduncularis transversus. Sollten aber alle Pupillenfasern die von Stili ing angegebene Bahn innehalten, so müssten alle Verletzungen des Vierhügels oberhalb der Sylvi'schen Wasserleitung auf die Pupillenweite ohne Einfluss bleiben. Es finden sich aber nun in der Literatur Angaben, dass die Verletzung der hinteren Hirncommissur die reflectorischen Pupillenbewegungen verändert. So hat z. B. Darkschewitsch ${ }^{15}$ ) nach vollständiger Zerstörung der hinteren Commissur des Kaninchens (Nr. 2) eine maximale Erweiterung beider Pupillen und vollkommene Aufhebung der Vermittelung des Lichtreizes auf die Oculomotoriuskerne beobachtet. Ausserdem findet er, dass die absteigende Wurzel Stilling's in keiner Beziehung zum Opticus steht, da die Fasern dieses Systems eine Myelinbekleidung bei Embryonen schon in einer solchen Periode besitzen, in welcher die Opticusfasern noch keine Spur davon enthalten. In seiner Dissertation ist Darksehewitseh auf Grund seiner histologisehen Untersuchungen zu dem Schluss gelangt, dass die Fasern des Tract. opt., welche in dem obersten Abschnitt des Pulvinars verlaufen und zu den Fasern der Pedunculi conorii sich hinzugesellen, als Pupillenfasern des Tract. opt. an'zusehen sind. Die Vermittelung des Lichtreizes von den Pupillenfasern im Tract. opt. zu dem Oculomotorius soll nach Darkschewitsch folgendermaassen vor sich gehen: Die Pupillenfasern sondern sich in der Gegend des lateralen Kniehöckers von der ganzen Masse der Fasern des Tract. opt. ab und streben in der Fórm eines zusammengedrückten Bündels schräg durch den hinteren Sehhügel- 
abschnitt zum Conariumschenkel hin. Nachdem sie sich zu der allgemeinen Masse des Conariumschenkels hinzugesellt, dringen die Pupillenfasern in die Substanz der Zirbeldrüse ein, woselbst sie auch enden. Aus der Zirbeldrüse entspringen die Fasern der hinteren Hirncommissur, welche nach der Kreuzung hinter der Sylvi'schen Wasserleitung in den oberen Oculomotoriuskern treten. An einer fortlaufenden Serie von Schnitten hat Darkschewitsch sich davon überzeugen können, dass alle zum Corp. genicul. lat. tretenden Fasern des Tract. opt. dieses Gebilde ausnahmslos nur mit einer dicken Schicht bedecken und theilweise auch seine Masse durchsetzen, aber durchaus nicht in seinen Zellen endigen. Auch im Pulvinar endigen sie nicht, sondern verlaufen an seiner Oberfläche. Weder der äussere Kniehöcker noch das Pulvinar bilden den Endigungsort für die Sehnervenfasern. Ohne auf die in seiner Dissertation genügend beleuchteten Ansichten für und wider diese Auffassung von Darkschewitsch hier weiter einzugehen, möchte ich nur noch einige spätere Daten über diesen Gegenstand doch berühren.

Ci o n ini ${ }^{25}$ ), welcher die Zirbeldrüse bei seinen Untersuchungen nach der Weigert'schen Methode färbte, glaubte zuerst, dass die dortselbst befindlichen Nervenfasern zu den Gefässen gehen, die Zellen aber sternförmige Bindegewebszellen darstellen. Hernach ${ }^{26}$ ) überzeugte er sich aber, dass die Gl. pinealis noch dünne, mit der hinteren Commissur und dem Oculomotoriuskern in Verbindung stehende Nervenfasern beherbergt, konnte aber dieselben nicht durch die ganze Drüse hindurch verfolgen. Auf Grund seiner Untersuchungen an Gehirnen von Hatteria punctata und Anguis fragilis hält $\mathrm{Ci}$ on in $\mathrm{i}$ das Conarium für ein Rudiment des Sehnerven vom Parietalauge, welche Meinung auch durch Obersteiner ${ }^{20}$ ) vertreten wird. Es hat sich nämlich erwiesen, dass bei einigen Thieren (Saurier, Selachier) das sogenannte Parietalauge mit der Zirbeldrüse durch einen Nervenstrang in Verbindung steht. Nach Eding er ${ }^{27}$ ) besteht die Gl. pinealis "weșentlich aus soliden Epithelschläuchen". Dagegen meint Bechterew, dass neueren Untersuchungen zufolge die Gl. pinealis ein Rudiment des dritten oder Parietalauges repräsentire und Nervenfasern enthalte ${ }^{5}$ ). Nach den neuesten Datén von Ramon ${ }^{21}$ ) gehe aus den eine Commissur der Gangl. habenulae darstellenden Pedunculi conarii keine einzige Faser in das Conarium über. Innerhalb dieses Organs selber sei aber eine ungewöhnliche Menge sich verästelnder Nervenfasern vorhanden, welche einen Plexus bilden, 
der an solche in wirklichen Drüsen erinnere. An der Basis des Organs stehe dieser Plexus mit einem reichen, die Hirnarterien umflechtenden Gefässplexus in Verbindung. Nach den Untersuchungen von Koelliker ${ }^{21}$ ) aber sind im Conarium der Katze und des Kaninchens eine geringe Anzahl Nervenfasern vorhanden, welche aus der Commissar der. Zirbeldrüsenschenkel stammen. Ferner hat Mendel ${ }^{28}$ ) die Existenz der Pupillenfasern im Gangl. habenulae und in der Commissura cerebri post. bestätigt, Gerlia ${ }^{29}$ ) aber die des oberen Oculomotoriuskernes und die Endigung des ventralen Theiles der hinteren Commissur im letzteren.

Bechterew ${ }^{8}$ ) dagegen findet, dass der Darkschewitsch'sche Kern einen Kern der hinteren Commissur darstellt, und dass in demselben die Fasern dieser Commissur und des hinteren Längsbündels eine Unterbrechung erfahren. Mit dem Oculomotorius stehe derselbe in keiner Beziehung. Die gut entwickelte hintere Commissur beim Maulwurf lässt sich nicht ganz gut mit ihrer Bedeutung als den Ort der Pupillenfasern vereinigen. Bechterew hat ferner am Maulwurfhirn ${ }^{5}$ ) einen kleinen Schenkel des Gl. pinealis nachgewiesen. Derselbe erfährt unter der Zirbeldrüse eine Kreuzung, richtet sich nach hinten und verliert sich bald darauf im medialen Theile des vordern Zweihügels. Auch $\mathrm{Ko}$ ellik er ${ }^{21}$ ) gelangt in Bezug auf den oberen Oculomotoriuskern von Darkschewitsch zum Schluss, dass derselbe mit der Commiss. cerebri post. in Verbindung steht und nicht zu den Ursprungskernen des Oculomotorius gehört, wesshalb er ihn als tiefen Kern der Commissura distalis bezeichnet. Nach Obersteiner $r^{20}$ ) ist es ebenfalls sehr wahrscheinlich, dass dieser Kern mit dem Oculomotorius in keiner Beziehung steht, und im Gegentheil mit der hinteren Commissur und mit dem hinteren Längsbündel zusammenhängt. Etwas später sind noch andere Ansichten über' die Bahn der Pupillenfasern zu den Oculomotoriuskernen aufgetaucht. So fand Mendel ${ }^{28}$ ) nach der Entfernung der Iris bei neugeborenen Thieren (Kaninchen, Katze, Hund) bei der Untersuchung des Gehirns Atrophie des Gangl. habenulae, des angrenzenden Theiles der hinteren Commissur und des Gudden'schen Oculomotoriuskernes, wesshalb er folgende Bahn für die Pupillenverengerung annimmt: Nerv. opt., Tract. opt., Gangl. habenulae, hint. Commissur, G u d d en 'scher Kern und der Oculomotoriusstamm. In den Vierhügeln und im Corp. genic. lat. hat er keine Veränderungen angetroffen. Perlia ${ }^{29}$ ) entfernte das eine Auge beim Kaninchen 
und untersuchte hernach das Gehirn dieser Thiere an Schnitten, welche nach Weigert und Marchi gefärbt worden waren. Hierbei gelang es ihm, einen Nervenzweig zu verfolgen, welcher vom Tract. pedune. transversus sich absondert, den inneren Rand des Grosshirnschenkels umbiegt und zu den anliegenden vorderen Wurzeln und Centren des Oculomotorius geht. Die Comm. post. und die radialen Fasern Meynert's waren nicht degenerirt. Perli a glaubt, dass der Peduncul. tranversus, welcher seiner Meinung nach vom Tract. opt. im vorderen Vierhügel entspringt, eben die Bahn darstellt, auf welcher die Reizung vom Opticus dem Oculomotorius übermittelt wird. (Gudde $\mathrm{n}^{30}$ ) hat festgestellt, dass nach der Enucleation des einen Auges der von Gall und Sporzheim¹) beschriebene Tract. pedune. transversus bei Kaninchen einer vollständigen Atrophie an der entgegengesetzten Seite unterliegt. Bei Katzen und Hunden ist die Atrophie dieses Gebildes beiderseitig, an der contralateralen Seite jedoch stärker. Der Nachweis des Zusammenhanges des Tract. pedunc. transversus mit den Oeulomotoriuskernen ist Gudden aber nicht gelungen. Auch Ganseris), Monakow ${ }^{33}$ ), Singer und Münzer ${ }^{34}$ ) haben der Zusammenhang des Tract. pedunc. transversus mit dem Auge behauptet. Nach Krause ${ }^{35}$ ) nimmt der Tract. pedunc. transversus bei Kaninchen seinen Anfang von der Subst. perforata media und endet im Tubere. post. thalami opt. und führt Sehfasern dem verlängerten Marke zu. „Nach der Meinung Anderer soll er sich mit den hinteren, sensiblen Oculomotoriuswurzel verbinden, was jedoch fraglich ist." Nach Adelheim ${ }^{36}$ ) tritt der Tract. pedunc. transversus im mittleren Drittel des vorderen Vierhügelrandes auf, geht hinter den medialen Kniehöcker, biegt um den Grosshirnschenkel und versenkt sich am medialen Rande des letzteren, etwas vor dem Abgangsort des Oculomotorius, in die Hirnmasse. $\mathrm{Zu}$ den Kernen dieses Bündels żählt A delheim einen Kern, welche zwischen den auseinandergehenden Fasern des an der Basis in die Hirnmasse eintretenden Tract. pedunc. transversus liegt. Bei der Atrophie dieses Bündels verschwinden auch die Zellen dieses Kernes. Zuletzt bemerkt Ade Iheim: „Ich habe unmittelbar vor dem vorderen Rande des Corp. quadrigem. ant. Zellen gesehen, welcher zu den Fasern des in Rede stehenden Bündels in Beziehung zu stehen scheinen, doch habe ich weitere Beweise zur Bestätigung dieses Verhältnisses bei meinen Untersuchungen nicht erhalten können." Beim Igel, welcher keine 
starken Sehnerven besitzt, ist der Tract. pedunc. transversus schwach, beim Maulwurf und Spolax typhlus, wie es scheint, gar nicht entwickelt. Bezüglich des Tract. pedunc. transversus meint $\mathrm{K}$ o ell $\mathrm{i} \mathrm{ke}^{21}$ ), dass weder sein Anfang noch sein Ende, trotz aller Mühe Gudden 's, bekannt geworden sei, und sein Centrum, das eigentlich kein Sehcentrum ist, sich im vorderen. Vierhügel befinde. Beim Kaninchen ist es Koelliker gelungen, sein Centrum oder seinen Ursprungsort aufzufiuden. Ausser dem schon $\mathrm{Gudden}$ bekannten Hauptbündel habe es aber noch zwei Nebenbündel, so dass im Ganzen drei Tract. pedunc. transversus vorhanden seien. Zweifellos befinde sich der Anfang des Tract. pedunc. transversus an der ventralen Hirnseite, und der im vorderen Vierhügel sich verlierende Streifen stelle sein Ende dar. Koelliker ist der Ansicht, dass dieses Ende in den tiefen Theilen des vorderen Vierhügels sich befindet. Die im Hauptbündel an der ventralen Hirnseite vorhandenen grossen, länglichen Zellen hält er dagegen für Ursprungszellen dieses Bündels. . B echterew ${ }^{5}$ ) beschreibt in der Tiefe der Grosshirnschenkel einen konischen, zwischen dem rothen Kern und der Subst. nigra gelegenen Kern, welcher Fasern vom Querbündel des Grosshirnschenkels aufnimmt, und nennt ihn Nucleus tract. pedunc. transversus. Hiermit sind jedoch die Voraussetzungen über die Bahnen der Pupillenfasern keineswegs erschöpft.

Bogrow ${ }^{37}$ ) glaubt, dass der Pupillenreflex, wenigstens theilweise, durch eine besondere, zuerst von Flechsig ${ }^{38}$ ) beschriebene Wurzel des Sehnerven, welche in den Tract. opt. von der Sehhügelbasis her eindringt und den Oculomotorius durch die hintere Commissur erreicht, vermittelt werde. Wie oben erwähnt, nimmt Gu d d en an, dass das Corp. genic. lat. das Centrum für die Pupillenfasern bilde. Hen schen dagegen behauptet, dass die Pupillenfasern : nicht in den äusseren Kniehöcker dringen, sondern vom Tract. opt. wahrscheinlich zwischen dem Grosshirnschenkel und dem äusseren Kniehöcker in medianer Richtung abgehen und in der vorderen Erhebung des Vierhügels endigen. Die Pupillenfasern besitzen seiner Meinung nach ihre gangliösen Zellen in der Netzhaut. Nach der Meinung Monakow's stammen die dicken Opticusfasern ebenfalls aus den Retinazellen. Doch sind andere Untersucher nicht dieser Ansicht. So nimmt $\operatorname{Ramon}^{21}$ ) z. B. an, dass die von-ihm gefundenen freien Endigungen in der Retina eben diejenigen empfindlichen Elemente seien, welche dem Gehirn die Itensität des Lichtes 
übermitteln und die reflectorische Wirkung auf den Sphineter pupillae bedingen. Pribytko ${ }^{10}$ ) schliesst aus seinen Versuchen mit Enucleation des einen oder beider Augen bei neugeborenen Thieren und besonders aus den Versuchen mit Enucleation des einen Auges bei erwachsenen Thieren (mit nachfolgender Bearbeitung des Präparates nach der Marchi'sehen Methode), dass die Sehnervenfasern zweifellos in der vorderen Erhebung des Vierhügels, im Corp. genic. lat, in den Tegmentzellen, zwischen der Subst. nigra und der Subst. reticularis, und vielleicht im Pulvinar endigen. Nach der Enucleation des einen Auges bei erwachsenen Kaninchen und Meerschweinchen beobachtete Pribytk ow Degeneration des entgegengesetzten Tract. pedunc. transversus, welche Degeneration wegen ihrer Beiderseitigkeit bei Katzen und Hunden weniger bemerkbar ist. Also bestätigt Pribytkow keine der angeführten Meinungen über den Verlauf der Pupillenfasern. Bechterew nimmt gegenwärtig an, dass die Pupillenfasern eine Strecke weit im Tract. opt. verlaufen, jedoch vor dem äusseren Kniehöcker sich nach innen wenden, indem sie ihrem Centrum resp. einem der Oculomotoriuskerne zustreben, wobei sie auf diesem Wege auf's Neue einer unvollständigen Kreuzung unterliegen ${ }^{5}$ ). Nach den neuesten Untersuchungen von Massaut ${ }^{39}$ ) mit Entfernung der ganzen Iris bei Kaninchen, nachheriger Tödtung des Versuchsthieres und Bearbeitung des Gehirns nach Marchi verlaufen die Pupillenfasern 1) im unteren Theil der hinteren Commissur und in dem inneren Abschnitte der von hier nach unten auseinandergehenden Fasern, 2) im vorderen Theil der tiefen weissen Schicht des vorderen Vierhügels, wohin sie aus der oberen weissen Schicht dieses Gebildes gelangen, und 3) im Tract. pedunc. transversus. Das Gangl. habenulae und der Gudden'sehe Kern waren -nicht degenirt. Die Abbildungen von Massaut erscheinen mir aber nicht genug überzeugend, weil eine und dieselbe Menge der schwarzen Punkte bald als normal, bald als pathologisch angegeben wird (vgl. Taf. VI, Fig. $3 D$ u. B). Solche geringe Veränderungen könnten wohl auch der Technik zur Last gelegot werden.

Genauere Daten als die bisherigen über den wirklichen Anfang und die thatsächliche Endigung der Pupillennerven sind meist an der Hand der Golgi'sehen Methode erhalten worden. Von den höheren Wirbelthieren sind die VögeI, bei welchen dem vorderen Zweihügel der Lobus opt. entspricht, am meisten studirt worden.

Santiago Ramon y Caja ${ }^{21}$ ) fand, dass der grösste Theil des 
Beiträge zur Erforschung des Sympathicuseinflusses anf die contral. Pupille. 209

Sehnerven im Lobus opt. mit complicirten und vollkommen freien Verzweigungen pinselförmig endet. Der Opticus enthält Axencylinder, welche aus den Zellen des Tect. opt. hervorgehen. Diese Fasern enden, wie es scheint, in der Netzhaut mit freien Endzweigen. In der grauen Substanz der Tect. opt. trifft man zahlreiche spindelförmige Nervenzellen an, deren Protoplasmafortsätze mit den freien Endverzweigungen der von der Netzhaut kommenden Fasern in Contact gerathen. Diese Beobachtungen sind von seinem Bruder Pedroc1), van Gehuchten ${ }^{40}$ ) und Koelliker ${ }^{21}$ ) bestätigt worden. Aus diesen Untersuchungen geht die wichtige physiologische Thatsache hervor, dass die centripetalen Fasern in der Gegend des Lob. opt. durch ihre Endverzweigungen nicht auf den Körper der Ganglienzellen, sondern nur auf die Verzweigungen ihrer Fortsätze einwirken; die Verbindung der Elemente unter einander kommt somit nur durch Contact zu Stande, d. h. es gilt hier, wie auch im Riechorgan, dass die Axencylinderfortsätze auf die protaplasmatischen und durch diese auf die Zelle einwirken.

Bei noch höheren Wirbelthieren hat Ko elliker ${ }^{21}$ ) bei der Maus pinselförmige Nervenendigungen im Corp. quadrigem. ant., Corp. genicul. ext. und Thalamus opt. vorgefunden. Besonders reich sind diese Endigungen im vorderen Vierhügel vorhanden. In den anderen Gebilden gleichen die Opticusendigungen einander und befinden sich in den inneren Theilen des äusseren Kniehöckers und an der dorsalen Thalamusfläche. Diese Endigungen sind von Koelliker in Fig. 696 und 697 abgebildet. Er bemerkt jedoch, dass er dünne Fäden ohne knopfförmige. Enden gesehen habe. P. Ram on ${ }^{4 i}$ ) hat baumförmige Opticusendigungen im lateralen Kniehöcker neugeborener Katzen und Ratten gefunden; Edinger ${ }^{27}$ ) hat sie copirt. Im vorderen Vierhügel gelang es P. Ramon (und auch Tartuferi ${ }^{21}$ ) nicht, Opticusendigungen aufzufinden, aber er fand, dass hier Zellen vorhanden sind, welche ihrer Axencylinderfortsätze in den Tract. opt. senden. Bei S. Ramon finden wir die Angabe, dass er bei neugeborenen Katzen ausgebreitete varicöse Verzweigungen der optischen Fasern im Kniehöcker und im vorderen Vierhügel vorgefunden habe. In der letzten Zeit beschrieb S. Ramon ${ }^{42}$ ) eingehend die Endverzweigungen der zum Tract. opt. gehörigen Fasern im äusseren Kniehöcker, den Thalamuskernen und im vorderen Vierhügel bei Mäusen, Kaninchen und Katzen. Ausserdem soll die in den vorderen Vierhügel eindringende Faserschicht des Tract. opt. viele Collateralen 
abgeben, von welchen ein Theil in das Stratum prof. alb. ciner. (Tortuferi) hinuntersteigt. Diesen Collateralen schreibt Ram on y Cajal die Uebermittelung der optisch-musculären Reflexe zu, woran sich jedoch auch das hintere Längsbündel betheiligen soll. Viele der absteigenden Collateralen endigen auch im centralen Höhlengrau. Schliesslich hat Le onowa ${ }^{43}$ ) die Hirne von Kindern ohne oder mit atrophirten Augäpfeln studirt und dieselben mit den Gehirnen von Neugeborenen verglichen. Sie fand, dass die Kreuzung im Chiasma nicht eine totale ist. Die Opticusfasern gehen theilweise aus der Netzhaut hervor und endigen im äusseren Kniehöcker; theilweise nehmen sie ihren Anfang von der oberflächlichen grauen Substanz der vorderen Vierhügel. Die F orel'sche Commissur fehlte stets, muss also eine Rolle beim Sehact spielen. Die Gudd en 'sche Commissur, diejenige von Meynert, das Conarium, die Pedunculi conarii, das Gangl. habenulae, die hintere Commissur, der Oculomotoriuskern, der innere Kniehöcker, die hinteren Vierhügel und der L n y s'sche Körper waren normal. Im äusseren Kniehöcker der Katze hat Leonowa an nach Golgi bearbeiteten Präparaten keine baumförmigen Verzweigungen gefunden. Die Tractusfasern sollen hier blind und stumpf endigen. Baumartige Verzweigungen hat sie wohl an Präparaten von Prof. Martin gesehen, zweifelt aber, dass dieselben dem Tract. opt. angehören.

Somit wäre positiv die Endigung der im Tract. opt. verlaufenden Fasern im Lob. opt. der Vögel und im vorderen Vierhügel, äusseren Kniehöcker und im Thalamus bei Mäusen, Kaninchen und Katzen bewiesen.

Das wäre Alles, was die Literatur uns über den in Rede stehenden Gegenstand bietet. Bechter ew ${ }^{5}$ ) hat somit vollkommen Recht, wenn er behauptet, dass unser Wissen über den Verlauf der $\mathrm{Pu}$ pillenfasern hinter dem Chiasma im Tract. opt. noch nicht über Voraussetzungen hinweg gekommen ist, wesshalb wir von zukünftigen Untersuchungen schon genauere Kenntnisse zu erwarten haben.

Eigene Untersuchungen. Als Material zu meinen Studien über den Verlauf der Pupillenfasern und überhaupt über die Beziehungen der subcorticalen Centren zu den Opticusfasern benutzte ich hauptsächlich die mir nach physiologischen Versuchen überwiesenen Thiere. Bei zwei Kaninchen wendete ich das Verfahren von Marchi an. Nach der Enucleation des einen Auges wartete ich 36 resp. 37 Tage, bevor ich die Thiere tödtete, um sowohl die 
Waller'sche als auch die centripetale (Darkschewitsch 'sche ${ }^{44}$ ) Degeneration der im Opticus verlaufenden Fasern zu erhalten. Die auf solche Weise erhaltenen Präparate belehrten mich, dass eine charakteristische Degeneration zweifellos in der mittleren, weissen Vierhügelschicht (Ko elliker), im Corp. genicul. lat., Pulvinar, Thalamus und im Tract. pedunc. transversus der entgegengesetzten Seite vorhanden war. Schwarzgefärbte Markschollen finden sich auch auf der anderen Seite in diesen Gebilden (ausser im Tract. pedunc. transversus) aber in weit geringerer Menge. Auf Grund dieser Versuche kann ich behaupten, dass 1) die Gudden'sche Commissur, 2) die Opticuswurzel Bogrow's, 3) der Fasciculus tuberis cinerei seu Commissura hypothalami ant. keiner Atrophie unterliegen. Ein vom Tract. pedunc. transversus zu den Oculomotoriuskernen gehendes, degenerirtes Bündel habe ich ebenfalls nicht bemerken können. Ebenso fehlt die charakteristische Degeneration des Luys'schen Körpers, des Corp. genicul. int. und der Erhebungen des hinteren Vierhügels. Gleich Michel ${ }^{25}$ ) habe ich in der Meynert'schen Commissur an meinen Präparaten wohl degenerirte Markschollen, aber an beiden Seiten in gleich geringer Menge angetroffen, was jedoch wohl in der besonderen Zartheit dieser Fasern und in der nicht gehörig vorsichtigen Behandlung des Hirnes seine Erklärung finden mag. Diese Erscheinung können wir nicht für einen Beweis des Zusammenhanges der Meynert'schen Commissur mit dem Auge ansehen, weil 1) die Degeneration an beiden Seiten angetroffen wird, und 2) es feststeht, dass die M e y nert'sche Commissur thatsächlich in keiner Beziehung zum Tract. opt. und den Opticuskernen steht ${ }^{5}$ ). In Bezug auf die Rad. desc. Stilling's, das Gangl. habenulae und die hintere Commissur kann ich nichts Endgültiges angeben und möchte nur vermerken, dass ich im ventralen Theil der hinteren Commissur stark ausgeprägte Degeneration angetroffen habe, welche aber vielleicht doch durch die nicht genug vorsichtige Behandlung der untersuchten Gehirne zu Stande gekommen sein mag.

Bei den bisherigen Untersuchungen ist nur die Methode Weigert's und die von Golgi in Anwendung gewesen, und die Methoden von Ehrlich und Dogiel haben nur zur Färbung feinerer Objecte gedient. Doch in letzterer Zeit ist das Methylenblau auch bei Gehirnuntersuchungen in Verwendung genommen. Ich verfuhr im Allgemeinen nach den Vorschriften, welche Stud. Kastorski im Laboratorium von A. Dogiel ${ }^{46}$ ) befolgt hat. Nur habe ich zu- 
weilen anstatt des Rasirmessers eine Scheere gebraucht und mich nicht bemüht, besonders dünne Schnitte zu erhalten, da ich beim Färben von oben beleuchtete. Dieses Verfahren bietet den Vortheil, dass die gegenseitige Lage der Theile weniger versehoben und die Gewebe weniger stark lädirt werden, was für die Färbung, wenigstens beim Gehirn, von Wichtigkeit ist. Um den Verlauf der Fasern des Tractus opt. in der Gegend des äussern Kniehöckers, des Sehhügels und der vorderen Erhöhung des Vierhügels zu verfolgen, entfernte ich bei soeben getödteten Thieren (Katzen und Kaninchen) die Hirnhemisphären nebst Gewölbe und die dünne Gefässhaut, worauf ich mittels einer Scheere die oberflächlichen Schichten des Tract. opt., des Corp. genicul. ext., des hinteren Theiles vom Thalamus opt, des vorderen Bindearmes und des vorderen Vierhügels derart abtrennte, dass sie als ein zusammenhängendes Stück erhalten wurden. Dieses Präparat lag bei dem Färben mit dem Ependym nach oben. Nach der Fixation der Färbung in gesättigter, wässriger Lösung von pikrinsaurem Ammoniak wurden unter der Lupe die etwaigen Reste der Gefässhaut und von der ungefärbten Seite mittelst einer Scheere das überflüssige Gewebe bis zur gewünschten Dicke des Präparates entfernt. An solchen Präparaten ist zu sehen, dass die Fasern des Tractus opticus den äusseren Kniehöcker bedecken; gegen den hinteren Theil des Sehhügels hin sondert sich eine ziemlich breite Schicht dieser Fasern ab, zerfällt aber bald und vermischt sich mit den hier in die verschiedensten Richtungen verlaufenden Nerven. Der etwas mehr nach binten gelegene Abschnitt des Tractus opt. setzt sich schräg nach innen und hinten als vorderer Bindearm fort und reicht bis zum vorderen Rand des vorderen Vierhügels, unter welchem er sich eben verliert. An solchen Präparaten scheinen die Fasern des Stratum zonale in den Conariumschenkel nicht einzudringen. Bei Katzen und Kaninchen kreuzen sich die Fasern des vorderen Bindearms nicht bei ihrem Eintritt in den vorderen Vierhügel mit den auf der Oberfläche des letzteren liegenden und querverlaufenden Nervenfasern, welche an solchen Präparaten aus dem Winkel zwischen dem vorderen Bindearm und dem äusseren Abschnitt des vorderen Randes vom oberen Vierhügel hervorgehen. Die nähere Betrachtung ergibt, dass dieses Bündel bei Kaninchen den Tractus peduncularis transversus repräsentirt (Fig. 1). Schon im Niveau des äusseren Drittels des Vorderrandes des oberen Vierhügels fangen zwischen den Nervenfasern dieses Bündels sich ovale, bipolare, kleine 
Nervenzellen an zu zeigen. Im weiteren Abschnitte dieses Bündels zur Mittellinie hin nimmt die Zahl der Nervenzellen immer mehr und mehr zu, und in der Gegend gegenüber dem inneren Drittel vom vorderen Rande des oberen Vierhügels ist darin schon eine Unmasse von Nervenzellen vorbanden. Die äussere Peripherie dieses Ganglions liegt ganz auf den Fasern des vorderen Bindearms; es reicht aber mit seinen inneren Abschnitten jedoch auch etwas tiefer, so dass über den Zellen einige Bindearmfasern sichtbar werden. Dieses Ganglion wird durch eine zellenlose Schicht deutlich von dem mehr nach innen und etwas nach vorn liegenden Ganglion habenulae abgegrenzt. Dieser soeben beschriebene Kern stellt wohl die von Ad elheim beschriebenen ${ }^{36}$ ) Nervenzellen dar, von welchen er nicht angeben konnte; welcher Art die Beziehungen derselben zum Tractus peduncularis transversus wären. Die von uns angewendete Methode erwies sich als am bequemsten zur Aufklärung dieser Beziehungen. Bei sorgfältiger Durchsicht ähnlicher Präparate-erwies es sich nämlich, dass die Zellen dieses oberflächlichen Kernes (Fig. 2) meist oval sind, einen Nucleus mit 1 oder 2 Kernkörperchen und nur 2 Fortsätze, einer inneren und einer äusseren, besitzen. Seltener werden dreieckige oder sternförmige Zellen mit vielen Fortsätzen angetroffen. Je näher die Zellen zur Mittellinie liegen, desto häufiger weicht ihre Form von der ovalen ab. Bei sehr vielen Zellen ist ein Axencylinderfortsatz sehr leicht zu sehen; derselbe geht in der Form eines langen Fadens mit spindelförmigen Verdickungen nach aussen zum Winkel zwischen der vorderen Erhebung des vorderen Zweihügels und dem vorderen Bindearm hin. Wenn auch sehr selten, so traf ich doch auch Axencylinderfortsätze, welche gegen die Mittellinie hin gerichtet waren. Der dem Axencylinderfortsatz entgegengesetzte Zellfortsatz verliert sich nach der Art der protoplasmatischen Fortsätze gewöhnlich bald. Der vordere Theil der Fasern des Tractus peduncularis transversus biegt gleichsam in der Richtung zum Sehhügel hin ein. Gelingt es zuweilen, diese Fasern auf eine weitere Strecke hin zu verfolgen, so sieht man, wie so noch mehr einbiegen und zum vorderen Bindearm in der Nähe des hinteren Sehhügelabschnittes gehen, d. h. in den Tractus opt. übergehen. Mir däucht es übrigens, dass ein Theil des Tractus peduncularis transversus auch den Sehhügel selber erreicht; besonders weisen die Präparate von Katzen darauf hin. Bei diesen Thieren wird der vordere Bindearm fast an seiner ganzen Ausdehnung an der Oherfläche von 
einzelnen Nervenfasern gekreuzt, welche vom hinteren Sehhügelabsehnitt zu dem Winkel ziehen, wo an meinen Präparaten der Tract. peduncul. transv. sich zeigt, oder auch zum äusseren Rand des Bindearms. Es wäre noch zu erwähnen, dass der Tract. peduncul. transv. in diesem Theil bei Kaninchen und Katzen aus 2 oder sogar aus 3 Bündeln zu bestehen scheint. Meine Präparate erlauben mir, zu behaupten, dass in der in Rede stehenden Gegend der Tract. peduncul. transv, nicht aus dem Vierhügel seinen Anfang nimmt (vgl. Fig. 1). An denselben Präparaten von Katzen kamen mir zuweilen unter der Opticusfaserschicht (Stratum zonale) auf der Oberfäche des hinteren Sehhügelabschnittes grosse multipolare Nervenzellen zu Gesicht, welche ibren Axencylinderfortsatz in die Fasern des Tractus opt., in der Richtung nach aussen, schicken. An meinen Flächenpräparaten von der Oberfläche des vorderen Zweihügels trifft man zerstreute kleine Nervenzellen von runder Form und mit einem oder einigen Fortsätzen an; auf der Oberfläche dieser Zellen lässt sich zuweilen ein pericelluläres Netz mit bedeutenden Verdickungen an den Knotenpunkten erkennen (Fig. 3). In der Tiefe der Präparates gelang es mir zuweilen im vorderen Zweihügel eine baumförmige, aus dünnen, varicösen Fäden bestehende Verzweigung der Nervenfasern zu sehen. Jeder dieser varicösen Fäden endete mit einer Verdickung (Fig. 4). Solche Bäumchen liegen in der Richtung von vorn nach hinten, und ich halte sie für Endigungen der Fasern des Tractus opt. im vorderen Zweihügel. Solche Verzweigungen der Nervenfasern im vorderen Zweihügel sah ich nur, nachdem das Präparat einem Druck ausgesetzt war. Wegen der tiefen Lage dieser Gebilde konnte ich die Nerven, welchen sie angehören, nicht weit genug verfolgen. An Präparaten, welche mittelst sagittal durch die vorderen Zweihügel angelegter Schnitte gewonnen waren, und an welchen solche Gebilde bequemer zu untersuchen sind, ist es mir noch nicht gelungen, dieselben zu färben. Nichtsdestoweniger kann ich aber doch die Thatsache bestätigen, dass in den oberflächlichen Schichten des vorderen Zweihügels Nervenendigungen vorhanden sind, welche dem Tractus opt. angehören. An denselben Oberflächenpräparaten aus dem hinteren Sehhügelabschnitte lässt es sich beobachten, wie im Stratum zonale von den markhaltigen Nerven Collateralen unter rechtem Winkel abgehen. Zur Bestimmung der näheren Beziehungen der Fasern des Stratum zonale zum äusseren Kniehöcker und zu dem hinteren Sehhügel- 
abschnitte habe ich aus dem Gehirn kurz vorher getödteten Katzen mit einem scharfen Rasirmesser Schnitte angelegt. Das Rasirmesser war mit einer ebenso starken Methylenblaulösung, wie sie zum Färben in Anwendung gelangte, angefeuchtet, und die ganze Procedur ging an dem in der Lösung befindlichen Präparat vor sich. Die Sehnittrichtung war sowohl frontal wie sagittal und auch horizontal, die Schnitte selber aber nicht gar zu dünn, weil sie sonst an das Messer kleben, wonach eine mechanische Läsion derselben unvermeidlich wird. Sogar an horizontalen Schnitten gelingt es zuweilen, zu sehen, wie die Fasern des Tractus opt. in den äusseren Kniehöcker bogenförmig übergehen, welches Verhalten aber besonders deutlich an frontalen Schnitten durch den hinteren Abschnitt des Corp. genicul. ext. und des Sehhügels zu Tage tritt. An derartigen Präparaten sieht man, wie eine Menge markhaltiger Fasern des Tractus opt. bogenförmig in den äusseren Kniehöcker übergeht und hier endet (Fig. 5). Im Corpus genicul. ext. lassen sich zwei Arten von Nervenendigungen unterscheiden, von denen eine jede eigentlich die Verzweigung einer Nervenfaser darstellt, morphologisch aber von der anderen stark abweicht. Bei der einen Endigungsweise giebt die markhaltige Nervenfaser schon im Stratum zonale, oder nachdem sie in den äusseren Kniehöcker hinabgestiegen ist und ein bestimmtes Niveau erreicht hat, eine unter rechtem Winkel abgehende marklose Collaterale $a b$, welche nach kurzem Verlauf sich anfängt stark zu schlängeln und schlielslich mit einer birnenförmigen Verdickung endet. Die Nervenfaser selbst verliert später in der Tiefe ihr Mark und endet, sich schlängelnd, auf dieselbe Weise. An der Bildungsstelle der Endschlängelung bemerkt man meist noch einen unter rechtem Winkel abgehenden dünnen, varieösen, äusserst langen, ebenfalls mit einer Endverdickung endigenden Faden. Aber nicht eine jede Stratumfaser gibt eine Collaterale ab. Häufiger geschieht es, dass die markhaltige Faser selber, nachdem sie mehr in die Tiefe des Corp. genicul. ext. gedrungen, anfängt sich zu schlängeln, unter rechtem Winkel einen sehr dünnen, varicösen Faden abgibt und mit einer birnenförmigen Anschwellung endet. Diese zweifellos den Fasern des Tractus opt. angehörigen Fasernendigungen liegen, wie der Vergleich meiner Präparate mit Fig. 6 von Monakow ${ }^{47}$ ) lehrt, in der Gegend des dorsalen Abschnittes vom äusseren Kniehöcker und ebenso zwischen ihm und dem ventralen Abschnitte, unter allen Umständen aber nur im caudalen Theil dieser Gebilde. 
Die zweite Endigungsform im dorsalen Kern des äusseren Kniehöckers ist ungeschlängelt und gehört markhaltigen Nervenfasern an, welche sich gabelförmig (nicht dichotomisch) in 2 Fasern theilen. Letztere nehmen den Farbstoff nur an einzelnen zerstreuten Stellen gut auf, während die übrigen Strecken sich äusserst schwach färben, wesshalb eine solehe Faser wie gefleckt aussieht. Diese secundären Fasern sind ziemlich dick und enden meist nach Zurücklegung einer bedeutenden Strecke mit einer Verdickung (Fig. $5 e$ u. $f$ ). Diese letzteren Endigungen gehören den aus dem medialen Abschnitte stammenden Fasern an, wesshalb sie weder zu den Seh- noch zu den Pupillenfasern gezählt werden können. Leider babe ich nicht vermocht, ihre Zugehörigkeit zu diesen oder jenen Bahnen des centralen Nervensystems festzustellen. Die beiden soeben beschriebenen Endigungsformen werden im Corp. genicul. ext. in grosser Menge angetroffen; hin und wieder findet man sie auch im hinteren $\mathrm{Ab}$ schnitt des Sehhügels, wohin sie aber nur aus dem äusseren Kniehöcker gelangt sein müssen. In dem Verbreitungsgebiet beider Nervenendigungen stösst man auch auf verschieden geformte Nervenzellen.

Da dem Conarium nach Darkschewitseh bei der Pupillenbewegung eine wichtige Rolle zukommt, weil hier die aus der Netzhaut stammenden Fasern endigen sollen, so habe ich auch diesem Gebilde meine volle Aufmerksamkeit geschenkt.

Was die Untersuchungstechnik anbelangt, so wurden bei den kurz vorher getödteten Thieren die Hirnhemisphäre und das Gewölbe entfernt, die Tela chorioidea sup. in der Gegend des 3. Ventrikels gefasst, vorn durchschnitten und nach hinten gezerrt. Das Conarium wird hierbei (bei Katzen) gewöhnlich nicht abgerissen, sondern nur von dem dasselbe umflechtenden Gefässnetz befreit. Das auf diese Weise freigelegte Conarium wurde entweder für sich oder mit den angrenzenden Hirntheilen exstirpirt und mit Methylenblau, wie oben, in toto gefärbt und nach der Fixation der Färbung unter der Lupe von seiner. Hülle befreit, wobei die überflüssigen Gewebstheile, um dünnere Objekte zu erhalten, mittelst einer Scheere entfernt wurden. Zuweileı erhält man bei der Untersuchung derartiger Präparate vorzügliche Bilder. Die sich ausgezeichnet mit Methylenblau färbenden Zellen des Pl. pinealis sind ziemlich klein und mit einem Kern versehen, der hin und wieder ein Kernkörperchen erblicken lässt (Fig. 7). Die Form der Zellen ist mannigfaltig: rundlich, unregel- 
mässig, vieleckig, oval und bịnförmig: Zuweilen sieht man aus dem Zellkörper viele kurze, sehr dünne (protoplasmatische) Fortsätze abgehen; ausserdem besitzen die Zellen noch einen dicken Fortsatz (Axencylinderfortsatz), welcher Collateralen abgiebt und hernach selber sich zu theilen anfängt. Zuweilen erblickt man sogar die Endverzweigungen dieses Fortsatzes hierselbst im Conarium (Fig. 9, a). Die in grosser Zahl in das Conarium eintretenden Nervenfasern scheinen mir hauptsächlich aus den Conariumschenkeln und aus der Commissur zwischen denselben zu stammen (Fig. 6); von diesen Theilen aus zieht sich nämlich ein dickmaschiges Netz von Nervenfasern, welche, je näher zur Spitze, desto dünner werden. An der Basis des Conariums findet ein Faseraustausch zwischen den einzelnen Maschen statt. Im Conarium habe ich drei Endigungsformen angetroffen: 1. eine pericelluläre, 2. eine freie, büschelförmige und 3. eine weniger entwickelte, den Axencylinderfortsätzen der hierselbst befindlichen Zellen angehörende.

Auf der Oberfläche einiger Nervenzellen des Conariums (Fig. 7) sieht man zuweilen nicht besonders dünne, varicöse, den Zellkörper umflechtende Fäden, welche violett oder auch dunkler erscheinen, je nach der Färbung der stets helleren Zellen. Hin und wieder stösst man auf die mit einer knopfförnigen Anschwellung versehene. Endigung eines solchen Fadens auf der Zelloberfläche (Fig. 7, d). Von der Oberfläche des Conariums aus bemerkt man auch in der Tiefe des Präparates Knäuel aus sehr dünnen, mit Endanschwellungen versehenen Fäden (Fig. 8). Betrachtet man einen solchen Faden im Knäuel in verschiedenen Niveaus, so bemerkt man, dass er sich theilt, und dass auch die secundären Zweige dasselbe thun, und dass die Endfäden mit Verdickungen versehen sind. Eine noch genauere Vorstellung über diese Gebilde erhält man, weinn man während der sorgfältigen Betrachtung des Fadens in verschiedenen Niveaus ihn zugleich zeichnet. Auf diese Weise erkennt man leicht, wie die primäre marklose Faser, sich theilend und schlängelnd, einen wirklichen Knäuel bildet, aus welchem die mit Anschwellungen versehenen Endfäden nach allen Seiten hinausragen. Ich kann zur Zeit nicht mit Gewissheit behaupten, dass diese Endigungen den an der Oberfläche des Conariums befindlichen Fasern angehören; mir scheint es jedoch, dass das thatsächlich der Fall ist. Die dritte Endigungsform gehört den Axencylinderfortsätzen der im Conarium selber vorhandenen Zellen an. Man sieht, wie ein solcher Fortsatz in einiger 
Entfernung von der Zelle unter Abgabe einiger Collateralen in einige mit Endanschwellungen versehene Zweige zerfällt (Fig. 9, a). Meine anatomisch-histologischen Untersuchungen erlauben mir somit, folgende Schlussfolgerungen $\mathrm{zu}$ machen.

1. Ein Theil der Fasern des Tract. peduncul. transv, entspringt aus einem besonderen, auf dem vorderen Bindearm, dicht am vorderen Rande des vorderen Vierhügels gelegenen Ganglion.

2. An den Nervenzellen der oberen Fläche des vorderen Vierhügels lässt sich ein pericelluläres Geflecht nachweisen.

3. In den oberflächlichen Schichten des vorderen Vierhügels sind Nervenendigungen vorhanden, welche wahrscheinlich den Opticusfasern angehören.

4. In dem Corp. genicul. laterale endigen eine Menge im Tractus opt. verlaufender und aus der Peripherie stammender Nervenfasern.

5. Zwischen diesen (sub 4 erwähnten) Endigungen befinden sich die Endigungen anderer, aus dem medialen Hirnabschnitte stammenden Nervenfasern.

6. Die Oberfäche des Conariums (unter der Membr. propria) ist mit Nervenzellen besäet.

7. An den Nervenzellen des Conariums kann ein dieselben umflechtender Nervenfaden, welcher allem Anschein nach hierselbst auch mit einer knopfförmigen Verdickung endet, nachgewiesen werden.

8. Unter den Nervenzellen des Conariums befinden sich solehe, deren Axencylinderfortsatz dortselbst auch endet.

9. In der Tiefe der Marksubstanz der oberen Conariumfläche finden sich verzweigte Gebilde, welche nach dem herrsehenden Brauch als Nervenfaserendigungen angesehen werden müssen.

Während die vorliegende Arbeit gedruckt wurde, hatte Professor L. Darkschewitsch die Liebenswürdigkeit, mir noch eine Arbeit von S. Ramón y Cajal zukommen zu lassen ${ }^{42}$ ). Nach diesem Forscher, welcher nach der Golgi'schen Methode gearbeitet hat, ist das Conarium ein drüsiges Organ, und die in ihm vorhandenen Nerven sollen dem Sympathicus angehören und mit den Gefässen verlaufen. Aber auch er hat im Conarium Nervenfasern gesehen, welche in kurze, varicöse, baumförmige, an ihrem Ende mit Körnchen oder Anschwellungen versehene Zweige zerfielen. Seine Figur gleicht 
der meinigen (Fig. 9, $b$ ). Die Verzweigungen der Nervenfaser sollen nicht mit Zellen zusammenhängen, wesshalb auch hier, wie im Lob. opt. der Vögel, eine Verbindung der Faser mit der Zelle nur durch Contact bestehe.

Meine Präparate zeigen aber (Fig. 7, $c$ u. $d$ ), dass das nicht überall so ist, und man auch einen innigeren Zusammenhang der Nerven mit den Zellen beobachten kann, nämlich eine Umflechtung der Zelle von der Nervenfaser. Ferner sind im Conarium auch besondere, specifische, kleine, sphärische oder unregelmässig gestaltete Zellen vorhanden. Dieselben besitzen $2-4$ verschieden lange Fortsätze, welche an die protoplasmatischen Fortsätze der Nervenzellen erinnern und mit einem Knötchen oder einer rundlichen Anhäufung von Protoplasma endigen.

Einen Axencylinderfortsatz konnte Ramón niemals sehen. Meine Präparate zeigen aber, dass sehr viele Conariumzellen ausser einer Menge dünner und sich verzweigender (Fig. 7, c) Fortsätze noch einen verhältnissmässig sehr dicken und zuweilen Collateralen abgebenden aufweisen, welchen letzteren ich wohl doch nur als Axencylinderfortsatz ansehen kann.

Physiologisches. Der Sympathicuseinfluss auf die gleichseitige Pupille ist schon längst bekannt; auf den Einfluss desselben Nerven auf die contralaterale Pupille ist man aber nur in der allerletzten Zeit aufmerksam geworden. Erst 1893 hat Professor Joh. Dogiel nämlich bei seinen an Hunden, Katzen und Kaninchen angestellten Versuchen festgestellt, dass die Reizung des Kopfstumpfes vom Halssympathicus ${ }^{*}$ ) an der entsprechenden Seite Erweiterung, an der contralateralen Seite aber Verengerung der Pupille bewirkt ${ }^{48}$ ). Diese Erscheinung veranlasste ihn, einen Zusammenhang des gegebenen Sympathicus mit dem pupillenerweiterndem Centrum des einen und dem pupillenverengenden Centrum des anderen Auges anzunehmen, wobei er aber die Art der Verbindung nicht näher bestimmt hat ${ }^{49}$ ). Nachher haben sich mit diesem Gegenstand Schenk und $\mathrm{Fuss}^{50}$ ) beschäftigt. Sie führen diese Erscheinung ganz auf consensuelle Reaction zurück, da sie ausgeblieben sei, wenn

*) Der Kürze halber soll der Kopfstumpf des Halssympathicus fernerhin einfach als Sympathicus bezeichnet werden. 
- auch bei der Dogiel'schen Versuchsanordnung - dafür gesorgt wurde, dass in das Auge der gereizten Seite kein Licht fiel. In ihrer Ansicht bestärkt sie noch der Umstand, dass bei Kaninchen, deren Pupillen nach den Versuchen von $\mathrm{Knoll}^{13}$ ), S te in a ch ${ }^{16}$ ) u. A. keine konsensuelle Reaktion aufweisen, die Sympathicusreizung keine Pupillenverengerung an der contralateralen Seite gebe. Jedoch abermals von Joh. Dogiel an Hunden und Katzen angestellte Versuche ${ }^{51}$ ), bei welchen das gleichzeitige Auge bald bedeckt und bald unbedeckt blieb, überzeugten ihn, dass auch beim bedeckten gleichseitigen Auge eine, wenn auch etwas weniger ausgesprochene Verengerung der Pupille am anderen Auge auftritt. Dabei ist in allen vier angeführten Versuchen vor der Verengerung zuweilen eine kurzdauernde Erweiterung der contralateralen Pupille vermerkt worden. Bei der durch Atropin bewirkten Paralyse der Oculomotoriusendigungen im Sphincter pupillae des contralateralen Auges wird die in Rede stehende Verengerung nicht erhalten. Dogiel setzt voraus, dass diese Erscheinung eine ziemlich complicirte ist, und dass sich daran nicht allein der Opticus, Sympathicus und Oculomotorius, sondern auch andere Nerven, wie z. B. der Vagus und überhaupt alle sensiblen Nerven, betheiligen, oder vielmehr, dass die Pupillenreaction infolge verschiedenartiger Beziehungen aller erwähnten Nerven und des Einflusses dieser Beziehungen auf die Nervenendigungen in der Irismusculatur eintritt. Eine ophthalmoskopische Beobachtung an Katzen und Hunden, besonders aber an Albinokaninchen ergibt, dass die Retina- und Choroïdeagefässe bei der Sympaticusreizung sich gleimässig und gleichzeitig zusammenziehen. Hieraus folge, "falls die Beobachtung nicht Selbsttäuschung ist", dass diese vom Sympathicus bewirkten „Veränderungen der Pupillenweite nicht unmittelbar von einer Veränderung der Gefässlumina abhängen, sondern unter Betheiligung des speciellen Nerven- und des Muskelapparates vor sich gehen". Die soeben erwähnte Veränderung in der Gefässweite ist auch von anderen, bei den Versuchen von Dogiel anwesenden Aerzten gesehen worden. „Von der Richtigkeit dieser Behauptung habe auch ich mich überzeugen können," sagte in seiner Dissertation Elins on ${ }^{52}$ ), welcher mehrere Jahre hindurch die Augenkrankheiten unter der Leitung des so bekannten Ophthalmologen E. A d amäk studirt hat. Damit ist aber auch die ganze Literatur des in Rede stehenden Gegenstandes erschöpft. 
Meine Aufgabe war, das Zustandekommen der Verengerung der contralateralen Pupille bei der Sympathicusreizung möglichst klarzulegen.

Die Schwankungen der Pupillenweite hängen bekanntlich von einer Menge von Factoren $a b$, welche von einigen Forschern ${ }^{13}$ ) in physiologische und physikalische eingeteilt werden. $\mathrm{Zu}$ den ersteren gehören die reflektorische und associirte Bewegungen bewirkenden Momente, zu den letzteren die elastischen und hydraulisehen.

Findet das Experiment unter Curare-Anwendung statt, so weist ein positives Resultat schon darauf hin, dass die zur Beobachtung gelangende Erscheinung keine associirte Bewegung darstellt, da eine solche Versuchsanordnung Accommodation und Convergenz ausschliesst. In solchem Falle gilt es zu entscheiden: 1. Ob die Pupillenverengerung infolge der Sympathicusrejzung an der anderen Seite nicht durch die Elasticität der Irisgewebe bedingt? 2. $\mathrm{Ob}$ diese Erscheinung nicht durch die Veränderungen in der Gefässweite und des Blutdruckes in der Iris desselben Auges hervorgerufen wird?

Kommt aber die in Rede stehende Pupillenverengerung weder durch Association noch durch hydraulische Ursachen zu Stande, und ist sie auch nicht ein Product der Elasticität, so muss sie eine physiologische sein. Alsdann harren folgende Fragen der Lösung: 1. Liegt hier nicht eine consensuelle Reaction vor ist? Bei derselben würden jedoch die Bewegungen beider Pupillen gleich sein, während sie unter den gegebenen Bedingungen aber doch entgegengesetzt sind. 2. Findet eine von der consensuellen Reaction unabhängige Pupillenverengerung infolge der Sympathicusreizung der contralateralen Seite statt? 3. Ist das der Fall, so galt es festzustellen, ob die Pupillenverengerung nicht von den Veränderungen der Blutcirculation, des intraocularen Druckes etc. in dem Auge der gereizten Seite abhängt. 4. Oder ist die in Rede stehende Pupillenverengerung nicht durch die in demselben Auge unter dem Einfluss der Reizung stattfindenden Verändungen bedingt? Fällt das Resultat negativ aus, so muss festgestellt werden, auf welchen Bahnen die Uebermittelung der Reizung von dem Sympathicus auf das contralaterale Auge vor sich geht, d. h. ob sie durch die Anastomosen des Sympathicus mit anderen Nerven am Halse und Kopfe oder durch die Gefässgeflechte an das Carotis interna stattfindet. 5. Spielen die Hirnnerven hierbei eine Rolle, und wenn, welche von ihnen? 6. In welchen Centren ist die Ursache der in Rede stehenden Erscheinungen zu suchen? 
Vor der Beschreibung der zur Lösung dieser Frage angestellten Versuche verdient aber ein Umstand besondere Erwähnung.

Meine Vorgänger haben die Schwankungen der Pupillenweite ohne weitere Hülfsmittel einfach beobachtet. Hierbei können aber geringe Veränderungen in der Pupillenweite unbemerkt bleiben; wenigstens waren bei meinen Versuchen die gleichzeitigen Angaben zweier Beobachter über die Pupillenbewegungen eines und derselben Auges in vielen Fällen widersprechend. Aus diesem Grunde musste ich der Messung der Pupillenweite den Vorzug einräumen. Von den Messungsmethoden der Pupillenweite erhält man die genauesten Resultate bei der Anwendung der Photochoreographen von Bellärmin $0 \mathrm{w}^{53}$, welches Instrument ich aber nicht erhalteu konnte. Die übrigen, von Foll in ${ }^{54}$ ), Halmogram ${ }^{54}$ ), Olbers ${ }^{54}$ ), Fick ${ }^{54}$ ), Land olt ${ }^{54}$ ), Donder ers ${ }^{55}$ ), A rlt ${ }^{55}$ ), Fr. Franck u. A. angegebenen Methoden sind bei künstlicher Atmung etwas unbequem oder verlangen das Augenmaass, bieten also keinen Vorzug vor meinem Verfahren. Da mich hauptsächlich nur die Veränderungen in der Pupillenweite, nämlich ob unter den angegebenen Bedingungen ihre Verengreung oder Erweiterung erhalten wird, interessirten, so benutzte ich eine nicht besonders genaue, fü meine Zwecke aber genügende Methode: an dem gut fixirten Kopf des curaresirten Thieres wurde ein ringförmiges, Millimetertheilung tragendes Maass aus Papier derart befestigt, dass es dem Niveau der grössten Pupillenweite möglichst angepasst war, und mit einem Auge beobachtet.

Die Versuchsanordnung selbst war folgende: das chloroformirte, mit dem Bauche nach oben an ein Brett befestigte Thier (meist Katze) wurde tracheotomirt und seine Venen am Halse oder am Schenkel zur Injection von Curare-Lösung freigelegt. War das Thier aufgewacht, so wurde die wässerige Curare-Lösung ( $8 \mathrm{p} \mathrm{M})$ injicirt und, nachdem die Athembewegungen aufgehört hatten, künstliche Athmung eingeleitet. Hierauf wurde der Halssympathicus auspräparirt und derart durehschnitten, dass sein Kopfstumpf bequem gereizt werden konnte. Zur Reizung diente der Schlittenapparat Du Bois-Reymond's mit einem mittelgrossen Grenet'schen Element. Die Stromstärke ersieht man aus den Protokollen durch den in Centimetern angegebenen Abstand zwischen den Spiralen. Der Verschluss des Auges geschah mit der Hand, welche die Lider aneinander brachte, wobei jedoch jeder Druck auf den Augapfel vermieden wurde; zuweilen bediente ich mich hierzu der Pincette. 
Versuch 5. 11. November 1896. Eine $1400 \mathrm{~g}$ schwere Katze; chloroformirt; mit dem Bauche nach oben an ein Brett fixirt; tracheotomirt; die Vena femoralis dextra freigelegt. Aus der. Narkose aufgewacht erhielt sie 1,5 cc $8 \%$ wässrige Curare-Lösung in die Vene, und nach dem Aufhören der Athembewegungen wurde künstliche Athmung eingeleitet. Der rechte Sympathicus auspräparirt, durchschnitten und sein Kopfstumpf in Ligatur behufs Reizung mit dem Inductionsstrom gefasst.

\begin{tabular}{|c|c|c|c|c|}
\hline Zeitangaben & $\begin{array}{l}\text { Welcher } \\
\text { Nerv gereizt }\end{array}$ & 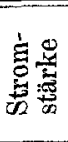 & 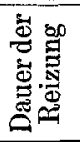 & $\begin{array}{l}\text { Untersuchungsgang und } \\
\text { Beobachtungseffect }\end{array}$ \\
\hline $1 \mathrm{~h} 50^{\prime}$ & . & & & $\begin{array}{l}\text { Die Pupillen reagiren auf das } \\
\text { Licht sowohl direct wie consensuell } \\
\text { äusserst träge. } \\
\text { Der Pupillenreflex an beiden } \\
\text { Augen deutlich und schnell. }\end{array}$ \\
\hline 2 h $10^{\prime}$ & & & & $\begin{array}{l}\text { Die Lider des rechten Auges } \\
\text { durch zwei kleire Pincetten, welche } \\
\text { das Haar erfasst hatten, geschlossen. } \\
\text { Der Lidschluss wird ausserdem mit } \\
\text { der Hand ca. } 1 / 2 \text { Minute vor jedem } \\
\text { Versuch und während desselben } \\
\text { verstärkt. }\end{array}$ \\
\hline Von 11--13' & & & & $\begin{array}{l}\text { Der Querdurchmesser der linken } \\
\text { Papille } 31 / 2 \mathrm{~mm} \text {. }\end{array}$ \\
\hline $13^{\prime}$ & $\begin{array}{l}\text { Reizung des } \\
\text { rechten Sympa- } \\
\text { thicus. }\end{array}$ & 15 & 50 & $\begin{array}{l}\text { Während der Reizung stellte sich } \\
\text { langsam eine Verengerung der linken } \\
\text { Papille von } 31 / \mathrm{mm} \text { bis auf } 3 \mathrm{~mm} \\
\text { ein und verschwand in } 12 \text { Secunden } \\
\text { nach dem Schluss der Reizung voll- } \\
\text { ständig. }\end{array}$ \\
\hline $\begin{array}{l}\text { Von } 14-15^{\prime} \\
30^{\prime \prime}\end{array}$ & & & & $\begin{array}{l}\text { Die Pupillenweite links die ganze } \\
\text { Zeit über } 31 / 2 \mathrm{~mm} \text { im Durchmesser. }\end{array}$ \\
\hline $15^{\prime} 30^{\prime \prime}$ & $\begin{array}{l}\text { Reizung des } \\
\text { rechten Sympa- } \\
\text { thicus. }\end{array}$ & 12 & 50 & 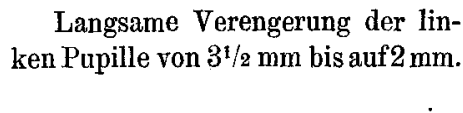 \\
\hline
\end{tabular}

Aus diesem Versuch geht hervor, dass auch beim curaresirten Thiere, bei welchem die Accomodation, die Convergenz und alle willkürlichen Bewegungen ausgeschlossen sind, eine Verengerung der Pupille auf Reizung des contralateralen Sympathicus eintritt. Folglich haben wir es hier nicht mit einer Associationserscheinung zu thun. Die Beobachtung lehrt, dass, angefangen von den Reptilien, die Pupillenweite beständig mehr oder weniger deutlich sehwankt ${ }^{16}$ ). 
Die von uns erhaltene Pupillenverengerung gehört auch nicht zu dieser Kategorie von Bewegungen, denn in der Zeitperiode, welche zwischen die Reizungen fällt, blieb die Pupille unbeweglich. An den Augen der höheren Thiere werden aber noch die sogenannten secundären Schwankungen in der Pupillenweite sichtbar: nach bedeutender Erweiterung oder Verengerung entsteht eine entgegengesetzte Bewegung der Pupille, woran sich immer schwächer werdende geringe Schwankungen der Pupillen, welche schliesslich mit blossem Auge kaum zu unterscheiden sind, anschliessen. Ruete ${ }^{16}$ ) erklärt die secundären Schwankungen dadurch, dass die Verengerung der Pupille eine Verminderung der in das Auge fallenden Lichtmenge bedingt, was dann eine Erweiterung der Pupille zur Folge hat; die hierdurch verstärkte Lichtmenge ruft wieder eine neue Pupillenverengerung herbei etc. Diese secundären Schwankungen können durch Elasticität des Irisgewebe erklärt werden. Dass wir in unserem Versuch es nicht mit diesen secundären Schwankungen zu thun hatten, kann man daraus ersehen, dass wir zuweilen sogleich eine Verengerung, ohne vorausgegangene Erweiterung, der Pupille erhielten. Ausserdem dauerte unsere Pupillenverengerung ziemlich lange, und in der grossen Mehrzahl der Fälle lassen sich hernach, auch sogar mit der Lupe, keine kleinen Schwankungen der Pupille nachweisen. In der Literaturübersicht haben wir erwähnt, dass Prof. Joh. Dogiel bei der Reizung des Kopfstumpfs vom Halssympathicus an beiden Augen von Kaninchen, Katzen und Hunden gleichzeitige Verengerung der Iris- und Choroideagefässe beobachtete. Durch eine solche Veränderung der Gefässlichtung müsste der Sympathicus weit eher eine Erweiterung als eine Verengerung der Pupille bewirken. Meine diesbezüglichen Versuche an Katzen, bei welchen zur bequemeren Beobachtung einen Monat vorher die äussere Irishälfte entfernt worden war, zeigten, dass die mit einem an dem Operationstisch befestigten Ophthalmoskop beobachteten kleinsten Retinagefäfse bei der Reizung des contralateralen Sympathicus mit starken Strömen wirklich verschwinden. Diesen Effect erhielt ich bei $8-6 \mathrm{~cm}$ Spiralenabstand. Ich kann ihm aber keine grosse Bedeutung beilegen, und zwar aus folgenden Gründen. Die Sympathicusreizung gibt im ersten Moment am contralateralen Ange zuweilen bekanntlich eine Erweiterung der Pupille. Am gleichseitigen Auge ist die Erweiterung, wie es schon lange bekannt ist ${ }^{56}$ ), von einem leichten Hervortreten des Augapfels begleitet. Es ist vorauszusetzen, dass die unter dem Einfluss des 
Sympathicus im ersten Moment erfolgende Erweiterung am anderen Auge ebenfalls mit Hervortreten des Augapfels verläuft, letzteres aber dem blossen Auge des Beobachters unsichtbar bleibt, nichtsdestoweniger aber die Entfernung zwischen dem Auge und dem Ophthalmoskopen verändert und hierdurch die feineren Netzhautgefässe versehwinden lässt. Zieht man ferner in Betracht, dass es eine ganze Theorie gibt, welche die Erweiterung der Pupille unter dem Einfluss des Sympathicus durch die Lichtungsveränderung der Irisgefässe zu Stande kommen lässt ${ }^{57}$ ), so wird man wohl kaum annehmen können, dass die Verengerung der contralateralen Pupille von Schwankungen der Gefässweite abhängt, da es doch unmöglich ist, dass derselbe Sympathicus die Gefässe in einem Auge contrahirt, im anderen aber dilatirt. Somit wird die in unserem Versuche erhaltene Verengerung der Pupille wohl kaum mit den Schwankungen der Lichtung der Irisgefässe im Zusammenhang stehen. Also muss ich auf Grund der in der Literatur vorhandenen Daten und auch eigener Versuche annehmen, dass die auf Reizung des contralateralen Sympathicus erfolgende Pupillenverengerung weder eine Associationserscheinung ist, noch durch die constanten Sehwankungen der Iris noch durch deren Elasticität noch durch die Veränderung ihres Blutgehaltes zu Stande kommt. Hieraus folgt, dass die in Rede stehende Veränderung der Pupillenweite das Resultat reflectorischer Thätigkeit ist. Diese Thätigkeit kann unter dem Einfluss des gereizten Sympathicus 1) im gleichseitigen, 2) im contralateralen und 3) in verschiedenen, mit dem gereizten Nerven direct oder indirect verbundenen Centren wachgerufen werden.

Wir haben erwähnt, dass Schenk und Fas die Pupillenverengerung in Folge der Reizung des contralateralen Sympathicus als consensuelle Reaction betrachten. Jedoch bemerkt Joh. Dogiel ${ }^{\text {r1 }}$ ) hierzu Folgendes. Fällt das Licht in die vorher vor ihm geschützt gewesenen Augen, so tritt bekanntlich Verengerung beider Pupillen auf. Es wäre zu erwarten, dass bei der bedeutenden, auf die Reizung des gleichseitigen Sympathicus erfolgten Erweiterung der Pupille auch diese letztere sich verengern müsste; hier tritt jedoch nicht Verengerung, sondern Erweiterung auf, und nur am contralateralen Auge bemerkt man eine Verengerung. Diese Erscheinung muss man nur dadurch erklären, dass der Einfluss des pupillenerweiternden Centrums stärker als die Wirkung des pupillenverengernden Nerven ist. 
Diese Voraussetzung kann man durch Versuche controliren. Bei der Reizung des Halssympathicus wirken ausser allen früheren Impulsen noch zwei neue auf die Pupille derselben Seite ein: 1) die Contraction des M. dilatator und 2) die hierdurch bewirkte grössere Lichtmenge auf die Netzhaut. Erscheint als Resultat aller dieser Einflüsse eine Erweiterung der Pupille, so folgt hieraus, dass die in das Auge fallende grössere Lichtmenge keine so starke Erregung des Oculomotoriuscentrums bewirkt, dass die Wirkung des Dilatators hierdurch gänzlich paralysirt würde. Vermehrt man aber z. B. durch eine biconvexe Linse die in das Auge fallende Lichtmenge, ohne die Reizung des Sympathicus, also auch die Wirkung des Dilatators, zu verstärken, so kann man durch die Zunahme der reflectorischen Erregung in den Oculomotoriuscentren auch bei der Reizung des gleichseitigen Halssympathicus Pupillenverengerung erhalteu. Von solchen Versuchen sei einer hier vorgeführt*).

Versuch 11. 30. December 1896. Ein $1540 \mathrm{~g}$ schwerer Kater. Die Vorbereitung zum Versuche wie früher. Curaresirt and künstliche Athmung eingeleitet. Zur Reizung wurde der Kopfstumpf des linken Halssympathicus in Ligatur gefasst. Beide Augen des Thieres während des Versuches unbedeckt. Ein sehr heller Tag.

\begin{tabular}{|c|c|c|c|c|}
\hline Zeit & Manipulationen & 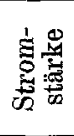 & 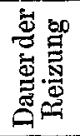 & Beobachtungsresultate \\
\hline $1 \mathrm{~h} 40^{\prime}$ & & & & $\begin{array}{l}\text { Die directe and die consensuelle } \\
\text { Pupillenreaction gut. }\end{array}$ \\
\hline$-43^{\prime}$ & $\begin{array}{l}\text { Reizung } d . \text { lin- } \\
\text { kenSympathicus } \\
\text { und gleichzeitige } \\
\text { Beleuchtung der } \\
\text { linken Pupillem. } \\
\text { einer Linse }{ }^{1 / 3} \\
\text { v. } 4 \text { Zoll Entfern. }\end{array}$ & 12 & 60 & $\begin{array}{l}\text { Die rechte Pupille verengerte } \\
\text { sich ziemlich schnell von } 13 / 4 \mathrm{~mm} \\
\text { bis auf } 1 / 3 \mathrm{~mm} \text {; die linke aber zeigte } \\
\text { eine geringe Erweiterung, welche in } \\
\text { eine Verengerung überging, worauf } \\
\text { einige Zeit hindurch Schwankungen } \\
\text { in der Pupillenweite stattfanden. }\end{array}$ \\
\hline$-46^{\prime}$ & Dito & 12 & 60 & $\begin{array}{l}\text { Die rechte Pupille verengerte } \\
\text { sich um ca. } 11 / 2 \mathrm{~mm} \text {. } \\
\text { Die linke Pupille verengerte sich } \\
\text { von } 11 / 3 \mathrm{~mm} \mathrm{bis} 1 / \mathrm{s} \mathrm{mm} \text {. }\end{array}$ \\
\hline
\end{tabular}

*) In allen Versuchen, in welchen beide Augen beobachtet wurden, lag die Beobachtung des Auges, dessen Schwankungen in mm angegeben sind, mir, die des anderen aber einem anderen (sehr erfahrenen) Untersucher ob. 
Beiträge zur Erforschung des Sympathicuseinflusses auf die contral. Pupille. 227

\begin{tabular}{|c|c|c|c|c|}
\hline Zeit & Manipulationen & 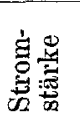 & 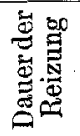 & Beobachtungsresultate \\
\hline $1 \mathrm{~h} 50^{\prime}$ & $\begin{array}{l}\text { Reizung d. lin- } \\
\text { kenSympathicus } \\
\text { und gleichzeitige } \\
\text { Beleuchtung der } \\
\text { linken Pupille m. } \\
\text { einer Linse }+1 / \mathrm{a} \\
\text { v. 4ZollEntfern. }\end{array}$ & 10 & 30 & $\begin{array}{l}\text { Die rechte Pupille verengerte sich } \\
\text { von } 11 / 2 \mathrm{~mm} \text { bís auf } 1 / 3 \mathrm{~mm} \text {; die } \\
\text { Verengerung trat während der Er- } \\
\text { weiterung der linken Pupille schär- } \\
\text { fer hervor. } \\
\text { Die linke Pupille gab anfänglich } \\
\text { eine Frweiterung und hierauf eine } \\
\text { Verengerung. }\end{array}$ \\
\hline$-55^{\prime}$ & Dito & 25 & 14 & $\begin{array}{l}\text { Beim Beginn der Reizung blieb } \\
\text { die linke Pupille unverändert, } \\
\text { während an der rechten eine Ver- } \\
\text { engerung von } 2^{1 / 2} \text { bis auf } 2 \mathrm{~mm} \text { be- } \\
\text { obachtet wurde; hernach links Er- } \\
\text { weiterung, rechts Verengerung von } \\
2 \mathrm{~mm} \text { auf } 1 / 2 \mathrm{~mm} \text {. }\end{array}$ \\
\hline$-58^{\prime}$ & Dito & 30 & 10 & $\begin{array}{l}\text { Rechts eine Verengerung von } \\
1 \mathrm{~mm} \text { bis zum vollständigen Schluss. } \\
\text { Links unbedeutende Erweiterung. }\end{array}$ \\
\hline$-59^{\prime}$ & Dito & 35 & 8 & $\begin{array}{l}\text { Linke Pupille kein Effect. } \\
\text { Rechts Verengerung von } 1 \mathrm{~mm} \\
\text { bis auf } 8 / \mathrm{s} \mathrm{mm} \text {. }\end{array}$ \\
\hline
\end{tabular}

Francois Franck ${ }^{58}$ ) hat schon vor uns bei der schwachen Reizung des Halssympathicus und gleichseitiger elektrischer Beleuchtung des entsprechenden Auges zuerst Verengerung und darauf stabile Erweiterung der Pupille erhalten. Der vorgeführte Versuch lehrt, dass bei stärkerer Frregung der Oculomotoriuscentren auch bei der Reizung des Halssympathicus an beiden Augen sogleich Pupillenverengerung eintritt. Hieraus folgt, dass trotz des scheinbar verschiedenen Charakters der Pupillenschwankungen die Verengerung der contralateralen Pupille, wenigstens theilweise, in der That durch consensuelle Reaction, wie Schenk und $\mathrm{Fass}^{50}$ ) behaupten, zu Stande kommt. Ist aber die ganze Verengerung der contralateralen Pupille auf die Rechnung der consensuellen Reaction zu setzen? Eine Reihe von Versuchen, bei welchen das entsprechende Auge während der Reizung des Sympathicus bedeckt blieb, erlaubt mir, zu behaupten, dass das nicht der Fall ist. Es erwies sieh, dass die Reizung des Halssympathicus bei bedecktem gleichseitigem Auge (zur Ausschliessung der consensuellen Reaction) unter gewissen Bedingungen und bei 
bestimmter Periode der Curarewirkung eine Verengerung der contralateralen Pupille bewirkt. Diese Verengerung betrug $1 / 3-1^{3 / 4} \mathrm{~mm}$, im Mittel $(16$ Beob. $)=1 \mathrm{~mm}$. Sind beide Augen unbedeckt, so erhält man unter gleichen Bedingungen etwas grössere Werthe. Als Beispiel möge folgender Versuch dienen.

Versuch 8. 21 November 1896. Eine $3800 \mathrm{~g}$ schwere Katze; zum Versuch wie früher vorbereitet; Curare; künstliche Athmung. Der Kopfstumpf des rechten Halssympathicus in ligatur gefasst. Es besteht weder directe noch consensuelle Lichtreaction der Pupille.

\begin{tabular}{|c|c|c|c|c|}
\hline Zeit & $\begin{array}{c}\text { Gang } \\
\text { der Untersuchung }\end{array}$ & 苞军 & 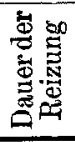 & Beobachtangsresultate \\
\hline $12 \mathrm{~h} 58^{\prime}$ & & & & $\begin{array}{l}\text { Die directe Lichtreaction } \\
\text { der Pupille träge. }\end{array}$ \\
\hline $1 \mathrm{~h} 2^{\prime}$ & & & & $\begin{array}{l}\text { Die directe und consen- } \\
\text { suelle Lichtreaction der Pupille } \\
\text { deutlich. }\end{array}$ \\
\hline & $\begin{array}{l}\text { Das rechte Auge } \\
\text { bedeckt. }\end{array}$ & $\begin{array}{l}\text { Von } \\
9-7\end{array}$ & 60 & $\begin{array}{l}\text { Am rechten Auge: Ver- } \\
\text { engerung von } 3 \mathrm{~mm} \text { bis auf } \\
1^{1 / 4} \mathrm{~mm} ; 10^{\prime \prime} \text { nach der Reizung } \\
\text { Erweiterung bis auf } 2 \text {, nach } \\
40^{\prime \prime} \text { bis auf } 2^{1 / 2} \text { und nach } 60^{\prime \prime} \\
\text { bis auf } 3 \mathrm{~mm} \text {; im Verlaufe der } \\
\text { nächsten Minute blieb die Pu- } \\
\text { pillenweite } 3 \mathrm{~mm} \text {. }\end{array}$ \\
\hline $1 \mathrm{~h} 7^{\prime}$ & $\begin{array}{l}\text { Reizung des rech- } \\
\text { ten Sympathicus. } \\
\text { Die linke Sympa- } \\
\text { thicus zur Reizung } \\
\text { vorbereitet. }\end{array}$ & & & \\
\hline $1 \mathrm{~h} 40^{\prime}$ & $\begin{array}{l}\text { Beide Augen unbe- } \\
\text { deckt. }\end{array}$ & & & \\
\hline$-42^{\prime}$ & $\begin{array}{l}\text { Reizung des rech- } \\
\text { ten Sympathicus. }\end{array}$ & 8 & 30 & $\begin{array}{l}\text { Die rechte Pupille maximal } \\
\text { erweitert. } \\
\text { An der linken Pupille eine } \\
\text { Verengerung von } 4 \mathrm{~mm} \text { bis } \\
\text { auf } 3^{1 / 2 .}\end{array}$ \\
\hline 1 h $52^{\prime}$ & $\begin{array}{l}\text { Reizung des linken } \\
\text { Sympathicus. }\end{array}$ & 12 & 60 & $\begin{array}{l}\text { Die linke Pupille maximal } \\
\text { erweitert. } \\
\text { Die rechte Pupille zeigt } \\
\text { eine Verengerung von } 11 / 2 \mathrm{~mm} \\
\text { bis zum vollk. Verschwinden. }\end{array}$ \\
\hline
\end{tabular}


Die Angabe von Schenk und Fuss, dass beim bedeckten gleichseitigen Auge die Verengerung der contralateralen Pupille ausbleibt, erschien mir zuerst äusserst seltsam, erklärte sich aber hernach ziemlich einfach. Meine Versuche belehrten mich, dass in gewissen Perioden der Curarewirkung weder directe noch consensuelle Reaction der Pupille besteht. Das direct in die Blutbahn geführte Curare wirkt ja zuerst auf das Gehirn ${ }^{59}$ ), wodurch eben bei bestimmter Dosis und in gewisser Wirkungsperiode dieses Giftes die Unbeweglichkeit der Pupillen bedingt wird.

Als ich anfing, mich vor der Reizung des Sympathicus davon zu überzeugen, wie weit die directe Lichtreaction ausgesprochen ist, stellte es sich heraus, dass eine Reizung des Sympathicus in der Zeit, wo die directe Lichtreaction abwesend ist, beim bedeckten gleichseitigen Auge, thatsächlich am contralateralen Auge effectlos bleibt. Dieser Umstand erklärt mir, wesshalb Schenk und Fuss bei ihren Versuchen an Kaninchen die in Rede stehende Erscheinung nicht erhalten haben.

Ich habe auch bei Kaninchen eine, wenn auch geringe (von $1 / 3-2 / 3 \mathrm{~mm}$ ) Verengerung der contralateren Pupille bei der Reizung des Sympathicus gesehen. Es sei hier ein solcher Versuch vorgeführt.

Versuch 10. 28. December 1896. Ein $1200 \mathrm{~g}$ schweres Kaninchen; vorbereitet wie die Katzen; 1 cc wässrige $8 \%$ Curare-Lösung unter die Haut; künstliche Athmung; beide Augen unbedeckt; beide Sympathici vorbereitet.

\begin{tabular}{|c|c|c|c|c|}
\hline Zeit & $\begin{array}{c}\text { Gang } \\
\text { der Untersuchung }\end{array}$ & 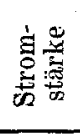 & 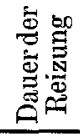 & Beobachtungsresultate \\
\hline $\begin{array}{c}\text { Von } \\
2 \mathrm{~h} 3^{\prime} \text { bis } 2 \mathrm{~h} 5^{\prime}\end{array}$ & & & & $\begin{array}{l}\text { Die rechte Pupille } 4 \mathrm{~mm} \\
\text { im Durchmesser. }\end{array}$ \\
\hline $2 \mathrm{~h} 5^{\prime}$ & $\begin{array}{l}\text { Reizung des linken } \\
\text { Sympathicus. }\end{array}$ & 12 & 40 & $\begin{array}{l}\text { Die rechte Pupille ver- } \\
\text { engerte sich von } 4 \mathrm{~mm} \text { bis auf } \\
3^{1 / 2} \mathrm{~mm} \text {; nach der Reizung er- } \\
\text { weiterte sich die Pupille wieder } \\
\text { bis auf } 4 \mathrm{~mm} \text {. } \\
\text { Am linken Auge: Pupillen- } \\
\text { erweiterung und starkes Her- } \\
\text { vortreten des Bulbus. }\end{array}$ \\
\hline
\end{tabular}




\begin{tabular}{|c|c|c|c|c|}
\hline Zeit & $\begin{array}{c}\text { Gang } \\
\text { der Untersuchung }\end{array}$ & 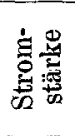 & 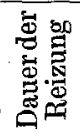 & Beobachtungsresuitate \\
\hline $2 \mathrm{~h} g^{\prime}$ & $\begin{array}{l}\text { Reizung des linken } \\
\text { Sympathicus. }\end{array}$ & 8 & 30 & $\begin{array}{l}\text { Rechte Pupille: Verenge- } \\
\text { rung von } 4^{1 / 2} \text { bis } 4 \mathrm{~mm} \text {. Linke } \\
\text { Pupille: Erweiterung und Her- } \\
\text { vortreten des Bulbus. }\end{array}$ \\
\hline $2 \mathrm{~h} 14^{\prime}$ & $\begin{array}{l}\text { Reizung des rech- } \\
\text { ten Sympathicus. }\end{array}$ & 7 & 30 & $\begin{array}{l}\text { Am rechten Auge Pupillen- } \\
\text { erweiterung und Hervortreten } \\
\text { des Bulbus. } \\
\text { Die linke Pupille verengert } \\
\text { sich von } 4 \mathrm{~mm} \text { bis auf } 33 / 4 \mathrm{~mm} \text {. }\end{array}$ \\
\hline 2 h $34^{\prime}$ & $\begin{array}{l}\text { Reizung des linken } \\
\text { Sympathicus. }\end{array}$ & 7 & 60 & $\begin{array}{l}\text { Die rechte Pupille verengert } \\
\text { sich von } 3 \mathrm{~mm} \text { bis auf } 3^{1 / 3} \mathrm{~mm} \text {. } \\
\text { Am rechten Auge Pupillen- } \\
\text { erweiterung. }\end{array}$ \\
\hline
\end{tabular}

Somit geht aus meinen Versuchen hervor, dass sowohl bei den Thieren mit consensueller Pupillenreaction (Katzen und Hunden) wie auch ohne solche (Kaninchen) ${ }^{*}$ ) eine Verengerung der contralateralen Pupille erhalten wird, welche bei Hunden und Katzen auch ohne Beleuchtung des anderen Auges eintritt. Sind aber beide Augen unbedeckt, so stellt sie sich bei letzteren Thieren 1) obne vorltergegangene Erweiterung, 2) schneller und 3) in verhältnissmässig stärkerem Grade ein.

Es könnte vorausgesetzt werden, dass unter dem Einfluss der schon von Serafino Biffi $1845^{55}$ ) nachgewiesenen Erweiterung der gleichseitigen Pupille in Folge der Sympathicusreizung die contralaterale Pupille sich zusammenzieht. Jedoch meine Versuche an Katzen, welchen in das gleichseitige Auge eine wässerige $3 \%$ salicylsaure Eserin-Lösung getropft worden war, haben gezeigt, dass eine Verengerung der contralateralen Pupille auch dann erhalten wird, wenn die gleichseitige ihre Grösse gar nicht verändert und als eine an beiden Enden verdickte Linie erscheint.

Ausser der Pupillenweite verändert sich im gleichseitigen Auge unter dem Einfluss der Sympathicusreizung noch der Blutdruck und der intraoculare Druck ${ }^{60}$ ). Sollten nun diese durchaus nicht indifferenten Veränderungen in dem einen Auge nicht am Ende den

*) Nach Knoll ${ }^{13}$ ) und steina $\mathrm{ch}^{16}$ ) und auch nach eigenen Versuchen an 6 Kaninchen muss diesen Thieren die consensuelle Reaction abgesprochen werden. 
Beiträge zur Erforschung des Sympathicusein flusses auf die contral. Pupille. 231

Grund für die Pupillenverengerung im anderen Auge abgeben? Um den Einfluss der Schwankungen des intraocularen Druckes in dem einen Auge auf die Pupillenweite des anderen Auges zu studiren, entfernte ich in zwei Versuchen vor der Sympathicusreizung ein Stück der Cornea und in einem Versuch ausserdem noch hernach die Linse aus dem gleichseitigen Auge. Alle diese Manipulationen blieben aber ohne Einfluss auf die Pupillenverengerung an dem contralateralen Auge. Selbstverständlich werden durch diese Operation die Schwankungen des intraocularen Druckes nicht ganz aufgehoben, sondern nur geschwächt.

Um endlich die Unabhängigkeit der Verengerung der contralateralen Pupille von allen möglichen, unter dem Einfluss der Sympathicusreizung erfolgenden Veränderungen im gleichseitigen Auge darzuthun, wurden einige Versuche mit vollständiger Enucleation des gleichseitigen Auges angestellt. Die nach einigen Minuten vorgenommene Sympathicusreizung gab aber dennoch Verengerung der contralateralen Pupille. Als Beispiel möge folgender Versuch dienen:

Versuch 7. 17. November 1896. Eine 2250 g schwere Katze; zum Versuch wie immer vorbereitet; Tracheotomie; Curare; kïnstliche Athmung. Enucleation des rechten Auges. Die linke Pupille wies 12 Minuten lang eine Grösse von $4^{1 / 2} \mathrm{~mm}$ im Durchmesser auf, erweiterte sich aber in dieser Zeit dreimal auf einige Secunden bis auf 7 und $8 \mathrm{~mm}$ und verengerte sich stets, aber nur bis auf $4^{1 / 2} \mathrm{~mm}$.

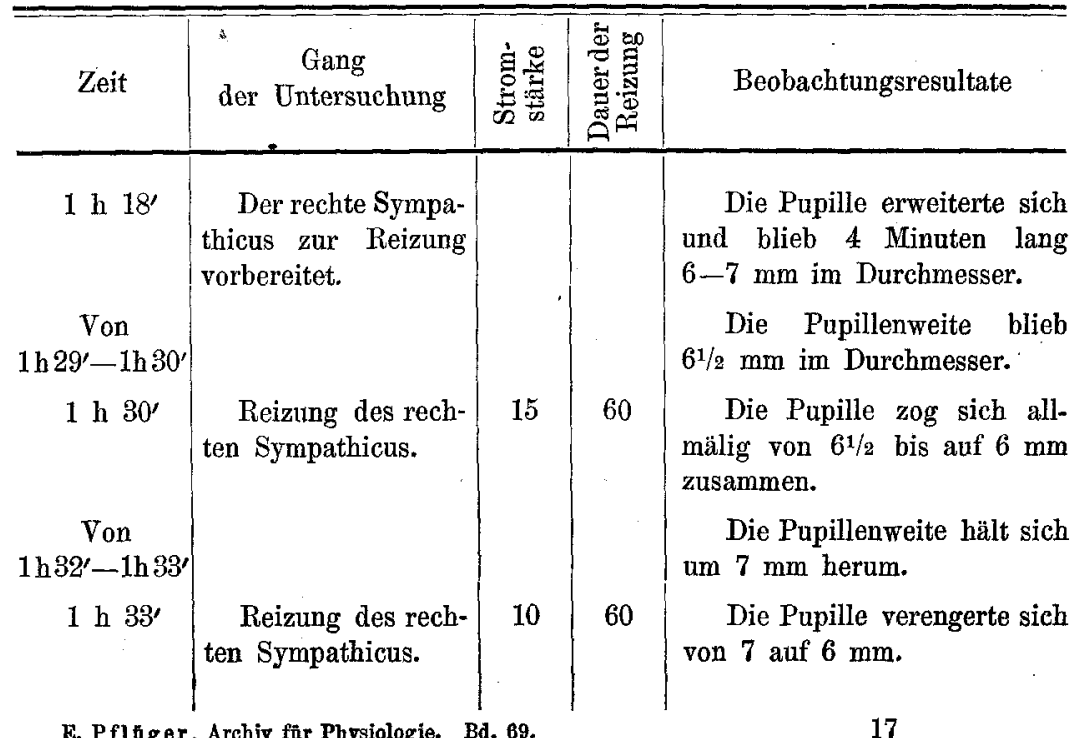

E. Pfl t̊ger, Archiv für Physiologie. Bd. 69. 


\begin{tabular}{|c|c|c|c|c|}
\hline , Zeit & $\begin{array}{c}\text { Gang } \\
\text { der Untersuchung }\end{array}$ & 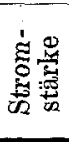 & 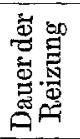 & Beobachtungsresultate \\
\hline $\begin{array}{c}\text { Von } \\
\operatorname{lh} 35^{\prime}-\ln 37^{\prime}\end{array}$ & & & & $\begin{array}{l}\text { Die Pupillenweite } 7 \mathrm{~mm} \text { im } \\
\text { Durchmesser. } \\
\text { Bei gleichbleibender Be- } \\
\text { leuchtung sind keine Schwan- } \\
\text { kungen der Pupillenweite be- } \\
\text { merkbar. }\end{array}$ \\
\hline $1 \mathrm{~h} 37^{\prime}$ & $\begin{array}{l}\text { Reizung des rech- } \\
\text { ten Sympathicus. }\end{array}$ & 10 & 60 & $\begin{array}{l}\text { Langsame Verengerung von } \\
7 \text { bis auf } 6^{1 / 2} \mathrm{~mm} \text {. }\end{array}$ \\
\hline
\end{tabular}

Derartige Versuche überzeugten mich davon, dass die Verengerung der contralateralen Pupille durch keine einzige Veränderung im diesseitigen Auge ausschliesslich bewirkt wird. Es frägt sich aber, ob die Verengerung der Pupille nicht durch Veränderungen in demselben Auge bedingt ist. So ist schon erwähnt worden, dass auf die Reizung des Sympathicus zuweilen eine Erweiterung der contralateralen Pupille eintritt (vor der Verengerung). Eine solche Erweiterung haben Schenk und Fuss und auch Prof. Joh. Dogiel gesehen. Ausserdem ist in diesem Auge von einigen Untersuchern noch eine Veränderung der Gefässlichtung beobachtet worden. Diese Erweiterung der contralateralen Pupille und Veränderung der Gefässlichtung ist von uns schon genügend erörtert worden (S.224 u. 225). Es könnten aber hierbei noch andere Einrichtungen mitwirken. Schon lange ist es bekannt, dass die Reizung verschiedener Bulbustheile am ausgeschnittenen Auge die Pupillenweite schwanken macht. In letzter Zeit hat Stein a $\mathrm{ch}^{{ }^{16}}$ ) an Katzen, Hunden und Kaninchen gefunden, dass die elektrische Reizung des enucleirten Augapfels Pupillenverengerung herbeiführt, und dass jeder deutlichen Verengerung regelmässig und unmittelbar eine schwache Erweiterung sich anschliesst, trotzdem die Reizung mit gleich starkem Strom fortdauert. Die nach solchen Schwankungen erreichte Grösse hält die Pupille bei, und nur nach der Einstellung der Reizung bekommt sie ihre vor dem Versuch gehabte Weite (S. 309).

Bei meinen Versuchen mit der Reizung des Sympathicus trugen die Schwankungen der Pupillenweite einen ganz anderen Charakter: im ersten Moment trat nicht immer eine Erweiterung ein; dann trat eine langsame Verengerung auf, welche zuweilen nach der Einstellung der Reizung noch zunahm; jedenfalls aber ging die Verengerung niemals während der Reizung in Erweiterung über. 
Beiträge zur Erforschung des Sympathicuseinflusses anf die contral. Pupille. 233

Könnte man in Bezug auf physiologische Erscheinungen den elektrischen Strom mit dem Nervenstrom identificiren, so wäre man schon nach der Gegenüberstellung der Pupillenschwankungen in beiden Fällen im Stande, zu entscheiden, dass die in Rede stehende Erscheinung nicht durch den Einfluss des gereizten Sympathicus auf die im contralateralen Auge befindlichen Apparate bedingt ist. Bei der gegenwärtigen Lage der Dinge bleibt uns zur Entscheidung dieser Frage nur ein Weg: Isolation des zu untersuchenden Auges von allen Apparaten, welche auf seine Pupille einwirken könnten, bei Erhaltung seines Zusammenhanges mit dem contralateralen Sympathicus mittelst der Gefässe. Dieser mühselige Versuch wird aher überflüssig, sobald es gelingt, irgendwie anders zum positiven Ergebniss zu kommen.

Alles bisher Angeführte drängt zur Voraussetzung, dass die in Rede stehende Pupillenverengerung eine reflectorische Erscheinung darstellt. Es bliebe uns also noch übrig, die Wege zu erforschen, auf welchen dieser Reflex übermittelt wird.

Es wäre wohl möglich, anzunehmen, dass die in Rede stehende Verengerung der Pupille durch den Einfluss des Sympathicus auf die von einigen Autoren angenommene Commissura arcuata anterior zu Stande kommt. In der Literatur finden sich Angaben, dass die sympathischen Fasern in den Opticus gelangen ${ }^{52}$ ). Meine Versuche an Hunden und Katzen mit Durchschneidung des Chiasma in der Mitte zeigten jedoch, dass nach dieser Operation die Pupillenverengerung ausbleibt. An das Chiasma gelangte ich von oben. Zur Orientierung diente mir die Kreuzungsstelle der Linien, welche längs der Mitte der von den Proc. zygomatici nach hinten und zur Mittellinie des Thieres ziehenden Erhöhungen gehen. Bei horizontaler Kopflage des Hundes befindet sich diese Stelle gerade über dem vorderen Rand des Chiasma. Jedoch nicht bei allen Hunderassen eignen sich diese Orientierungslinien. Bei Katzen befindet sich der vordere Chiasmarand in der Mittellinie $4 \mathrm{~mm}$ vor dem Punkte, der mir zur Orientirung bei der Durchschneidung der Nerven im Sinus cavernosus diente (vgl. S. 235). Der Schädel wurde trepanirt, der Sinus cavếrnosus an zwei Stellen unterbundien und durchschnitten. Die Blutung aus der Diploë wurde durch das Eindrücken einer Mischung von Vaselin und Wachs in die Knochenwunde gestillt. Das Messer (Graefe's Cataractmesser) wurde perpendiculär eingestochen und der Schnitt von vorn nach hinten geführt. Wenden wir uns jetzt 
zum Studium der Reflexbahnen, auf welchen die Reizung des einen Sympathicus auf die contralaterale Pupille verengernd wirkt.

Den Verlauf des Sympathicus, besonders in Verbindung mit den anderen Nerven, verfolgte ich an Katzen, welche schon zu den Versuchen gedient hatten. Nachdem das Präparat 5-24 Stunden in einer 1/2 procentigen Essigsäure-Lösung gelegen hatte, waren bei dunkler Unterlage die feinsten Nervenzweige sichtbar. Der Halssympathicus gibt oberhalb der Stelle, welche mir gewöhnlich zur Reizung diente, vor allen Dingen dünne Aeste zu den zum Wirbelcanal verlaufenden Halsnerven (vgl. Fig. 10) ab; noch höher hinauf bildet er das ovale, ziemlich bedeutende Ganglion cervicale superius. Aus den verschiedenen Abschnitten desselben gehen Anastomosen $a b: 1$. zum Plexus ganglioformis (eine kurze und dicke) wie beim Froseh ${ }^{61}$ ), 2. zum IX. und XII. Hirnnerven und ebenso zu den ersten zwei Halsnerven. Aus der vorderen Peripherie gehen fast horizontal 2-3 Aestchen zur Carotis comm., nämlich zu ihrer Theilungsstelle. Hier bilden diese $Z$ weige einen Plexus, aus welchen längs den verschiedenen Gefässen dünne Nervenfäden abgehen. Vom oberen Ende des oberen Halsknotens gehen 4-5 Nerven ab; unter ihnen sind einige ziemlich dick. Diese Nerven sind mit einander durch dünne Fäden verbunden und verlaufen circa $5 \mathrm{~mm}$ ganz frei, worauf das ganze Bündel durch einen besonderen Canal in die Schädelhöhle tritt, indem es in der Form eines flachen Bandes auf der medialen Fläche des inneren Abschnittes der Wand von der Bulla ossea liegt. Noch während des verticalen Verlaufes schicken zwei Nerven dieses Bündel’s dünne Fäden in der Pyramidengegend zur Anastomose mit den Hirnnerven. Ein Nerv des Bündels verliert sich hier ganz, indem er sich einem Hirnnerven hinzugesellt. Die Wand der Bulla ossea und den Verlauf der Nerven hier sieht man am besten, wenn man mittelst der Knochenscheere einen Theil der hinteren Pyramidenfläche vom Schläfenbein entfernt. Zu diesem Zweck wurde zuerst der obere Halsknoten und die von demselben nach oben gehenden Nerven abpräparirt, dann ein entsprechender Theil vom Schädel ausgebrochen, worauf die eine Wange der Knochenscheere fast auf den oberen Rand der Pyramide, die andere aber sogleich nach innen von den vom obern Halsknotenende zum Schädel gehenden Nerven gestellt wird. Man kann auch anders verfahren: Die weichen Theile unterhalb des Ohres werden bis auf den Knochen entfernt, wobei die untere Wand der Bulla ossea sichtbar wird. Um 
die Nerven zu sehen, müssen die Kammern der Bulla ossea und vorsichtig auch ihre untere und innere Wand entfernt werden. In der Schädelhöhle erscheinen diese Nerven im äussersten Abschnitt impressionis n. trigemini. Bei ihrem Austritt sind sie von einem Knochenvorsprung bedeckt, welcher über dem äusseren Theil des Ganglion Gasseri liegt. Hebt man ihn empor und nach aussen, so sieht man, wie die Nerven unter der das Ganglion Gasseri umhüllenden Membran in den Sinus cavernosus treten. Sie erscheinen somit nach aussen von der Carotis. Diese Zweige des Sympathicus anastomosiren oben mit dem Ganglion Gasseri, dem VI. und III. Hirnnerven. Ich habe eine Anastomose mit dem Trochlearis nicht auffinden können. Die Anastamose mit dem Oculomotorius ist ihrer Länge wegen nicht immer zu sehen. Zu dem Abducens gesellt sich der dickste Nerv, welcher dabei auch ganz verschwindet. Die übrigen Fäden des Sympathicus ziehen nach den angegebenen Anastomosen weiter und legen sich dem ersten Trigeminusaste nur an. Der weitere Verlauf derselben interessirte mich wenig, da es sich erwies, dass für die in Rede stehende Erscheinung hauptsächlich die schon angegebenen Abschnitte des Sympathicus von Bedeutung sind.

Um die Frage $\mathrm{zu}$ entscheiden, welche von diesen Verbindungszweigen die Reizung vom Sympathicus auf das contralaterale Auge übermitteln, habe ich eine Reihe von Durchschneidungen dieser Anastomosen vorgenommen. Isolirt man bei curaresirten Katzen den Sympathicus und den oberen Halsknoten von allen Aastomosen, mit Ausnahme nur der vom oberen Knotenende abgehenden Nerven, so erhält man dennoch, bei bedecktem gleichseitigem Auge, die Verengerung der contralateralen Pupille bei jeder Reizung des Halssympathicus.

Bei derartigen Versuchen isolirte ich den Halssympathicus, indem ich von vorn auf ihn eindrang und längs demselben nach oben ging. Zur Isolation des oberen Halsknotens kann man noch einen Hülfsschnitt seitlich am Halse machen, wobei das Thier auf der anderen Seite liegt. Jedenfalls muss der Ursprung des M. sterno-cleidomastoideus abgetrennt, der Plexus ganglioformis und ein Theil des Vagus oberhalb desselben ganz entfernt werden, weil sie den Sympathicus verdecken. $\mathrm{Zu}$ möglichst vollkommener Isolation wurden noch mittelst einer Scheere zwei Schnitte bis auf den Knochen vor und hinter den vom oberen Knotenende in den Schädel tretenden Nerven gemacht. Nach dem Versuch wurde die Operation durch Präpariren des untersuchten Gebietes in 1/2\% Essigsäurelösung controlirt. 
Versuch 27. 9. Februar 1897. Eine $1300 \mathrm{~g}$ schwere Katze; wie früher befestigt, tracheotomirt; 1,5 cc 8\% Unterbindung der Art. carot. comm. Entfernung des Plexus ganglioformis und eines Theiles des oberhalb befindlichen Vagus. Ausser den nach oben gehenden Zweigen alle Anastomosen des Sympathicus und des oberen Halsknotens durchschnitten. Sorgfältige nachherige Untersuchung des Präparates in $1 / 2 \%$ ige Essigsäure-Lösung.

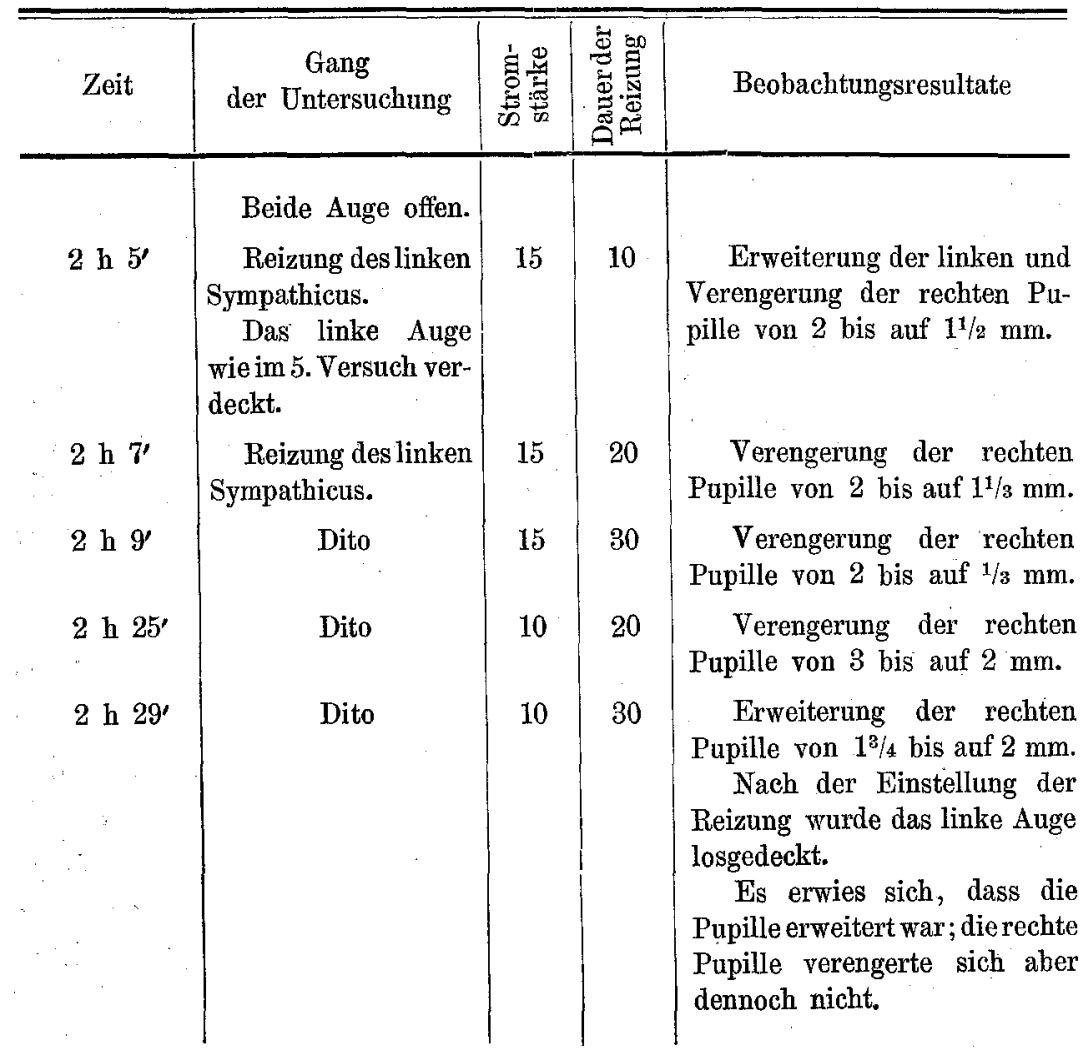

Nachdem so durch mehrere ähnliche Versuche es festgestellt war, dass bei der Verengerung der contralateralen Pupille die vom oberen Halsknoten abgehenden Nerven, welche durch das Schläfenbein treten oder eine Anastomose in der. Nähe dieses Knochens bilden, eine Rolle spielen, musste auf ihre Bedeutung näher eingegangen werden.

Anatomische Thatsachen zwingen uns, anzunehmen, dass die Kopfzweige des Sympathicus einen Einfluss auf das contralaterale Auge (bei bedecktem diesseitigem) nur durch Hirncentren ausüben können. Eine Verbindung dieser Zweige mit den Hirncentren kann 
durch die Anastomosen mit dem Oculomotorius, Trigeminus, Abducens etc. zu Stande kommen. Alle diese Anastomosen lassen sich in zwei Gruppen theilen: die eine liegt in der Schädelhöhle und ist dem Messer leicht zugänglich; die zweite liegt aber in der Dicke der Knochen oder so hoch (an dem Schädelknochen selber), dass eś sehr schwer ist, sich über ihre Lage bei lebenden Thieren zu orientiren und sie zu lädiren. Um zu entscheiden, durch welche von diesen Anastamosen die in Rede stehende Erscheinung zu Stande kommt, durchschnitt ich die im Sinus cavernosus verlaufenden Nerven. Den Sinus cavernosus erreichte ich von oben oder von der Seite. Im ersteren Falle diente mir zur Orientirung ein $5 \mathrm{~mm}$ starker Glasstab, dessen Ende in den äusseren Gehörgang geführt wurde. $4 \mathrm{~mm}$ vor dem vorderen Glasstabrande und $3,5 \mathrm{~mm}$ von der Mittellinie liegt die Stelle des Oculomotorius $2 \mathrm{~mm}$ hinter seinem Eintritt in den Sinus cavernosus. Hierbei muss der Kopf der Katze auf eine vollkommen horizontale Ebene derart placirt werden, dass die Linie von dem vorderen Nasenrande und von der oberen Lippe volkommen perpendikulär vom Horizont sind (die Lippe muss ein wenig vorstehen).

Fünf derartige Versuche ergaben folgendes Resultat: 1. Werden alle im Sinus cavernosus verlaufenden Nerven durchschnitten, so bleibt die Verengerung der contralateralen Pupille, bei der Sympaticusreizung und bedecktem gleichseitigem Auge aus; 2. bei verschiedenen Läsionen wird die Verengerung unter den angegebenen Bedingungen noch erhalten, falls der Oculomotorius nicht oder nur leicht lädirt ist; 3. bei vollständiger Durchtrennung des Oculomotorius der gereizten Seite bleibt die Verengerung der contralateralen Pupille bei bedecktem gleichseitigem Auge aus. Ausserdem ist zu erwähnen, dass nach der Verwundung der unteren Peripherie des ersten Trigeminusastes (und der dünnen an ihrer Peripherie verlaufenden Sympathicuszweige) die Reizung des Sympathicus an dem gleichzeitigen Auge nur Erweiterung der äusseren Hälfte der Iris gab, während die innere unbeweglich blieb.

Alle diese Versuche beweisen also, dass der. Einfluss des Sympathicus auf das entgegengesetzte Auge durch jene Fasergruppe vermittelt wird, welche im Sinus cavernosus verläuft, die übrigen Anastamosen mit den Hirnnerven hierbei als gleichgültig sind.

Um zu erfahren, welcher von den im Sinus cavernosus verlaufenden Nerven die sympathischen, zum Gehirncentrum gehenden Fasern 
enthält, habe ich weitere Durchschneidungsversuche vorgenommen. Da aus der Literatur bekannt ist, dass ein Theil der Sympathicuszweige sich zum Ganglion Gasseri hinzugesellt ${ }^{55}$ ) und mit dem ersten Trigeminusaste zum Auge gelangt (Baloch, Oehl etc.), so konnte angenommen werden, dass ein anderer Theil der Sympathicuszweige durch denselben Nerven zu den Gehirncentren geht. $\mathrm{Zu}$ diesem Zwecke habe ich einen Versuch mit Durchschneidung des Trigeminus zwischen dem Ganglion Gasseri und dem Gehirn gemacht. Nach einigen Uebungen an Katzencadavern ist diese Operation nicht schwer auszuführen. Man bedient sich hierbei eines stumpfspitzigen Messers, dessen scharfer Rand etwas eingebogen ist. Sogleich hinter dem äusseren Gehörgange der Katze bemerkt man eine dem Proc. mastoideus entsprechende Knochenerhabenheit, von welcher zur Protuberant. occip. extern. ein ziemlich deutlicher Knochenkamm zieht. Am letzteren wird, 7-8 $\mathrm{mm}$ von der Mittellinie, mit der Knochenscheere eine Oeffnung gemacht und nach innen zu, wenn nöthig, erweitert. Durch diese Oeffnung wirci das Messer längs der hinteren Fläche der Schläfenbeinpyramide bis zu einer schon vorher bestimmten Tiefe vorgeschoben und der Schnitt von vorn und oben nach hinten und etwas nach unten ausgeführt. Die Durchschneidung gab im ersten Moment eine Erweiterung und hierauf eine geringe, aber dauernde Verengerung der gleichzeitigen Pupille. Der Einfluss des Sympathicus auf die contralaterale Pupille blieb aber bestehen. Bei verdecktem gleichseitigem Auge gab die 30 Secunden dauernde Sympathicusreizung, bei $12 \mathrm{~cm}$ Spiralenabstand, jedes Mal eine Verengerung der contralateralen Pupille: von drei Versuchen betrug die Verengerung zweimal $1 \mathrm{~mm}$ (von $2 \mathrm{~mm}$ bis auf $1 \mathrm{~mm}$ ) und einmal $1 / 2 \mathrm{~mm}$ (von $1 \frac{1}{1} / 2 \mathrm{~mm}$ bis auf $1 \mathrm{~mm}$ ). Bei offenem gleichseitigem Auge rief eine solche Reizung eine Verengerung der contralateralen Pupille von $\mathbf{1}^{1 / 2} \mathrm{~mm}$ bis auf $1 \mathrm{~mm}$ hervor. Die nach dem Versuche vorgenommene Obduction ergab, dass der rechte Trigeminus zwischen der Varolsbrücke und dem Ganglion Gasseri vollständig durchschnitten war. Somit war es also klar, dass die in Betracht kommenden Sympathicuszweige die Hirncentren nicht durch den Trigeminus erreichen.

Jetzt wandte ich mich an den Oculomotorius. Er wurde in dem Raume zwischen dem Sinus cavernosus und seiner Ausgangsstelle aus dem Gehirn durchschnitten: fünfmal von oben her durch das ganze Gehirn hindurch und dreimal nach Erheben der 
Hemisphäre. Die erste Methode verlangt äusserste Genauigkeit der Kopfstellung des Thieres beim Einsenken des Messers. Die geringste Abweichung der Handhabe des Messers oder des Kopfes hat einen Schnitt an einer unrichtigen Stelle zur Folge. Die zweite Methode ist viel leichter und deshalb besser, wesshalb ich sie hier kurz angeben will.

Die Haut wird in der Mittellinie des Kopfes durchschnitten, das Pericranium längs der Ursprungsstelle des Schläfenmuskels ebenfalls, worauf die beiden letzteren von dem Knochen abgeschabt werden. Hierauf entfernt man mittelst einer Knochenscheere einen bedeutenden Theil der entsprechenden Hälfte des Schädeldaches, wobei die äussere Wand möglichst weit nach unten mitgenommen wird. Die Blutung aus der Diploë wird auf die angegebene Weise gestillt. Hierauf wird die harte Hirnhaut durchtrennt, ihre Lappen zur Seite gedrängt und unter die Hirnhemisphäre vorsichtig eine gebogene Platte geführt, mit welcher sie leicht emporgehoben wird. Mit einem stumpfspitzigen Messer geht man jetzt in die Schädelböhle vor der vorderen Wand des äusseren Gehörganges und schiebt es in der tiefsten Stelle des Schädelgrundes längs der harten Hirnhaut nach innen. In einer bestimmten Tiefe gleitet die Messerspitze längs der äusseren Wand des Sinus eavernosus nach oben. Sobald die Messerspitze die obere Grenze des Sinus cavernosus erreicht hat, wird das Messer nach vorne geführt, wo es bald auf den Oculomotorius bei seiner Eintrittsstelle in den Sinus cavernosus trifft. Den Moment, wo der Nerv durehschnitten wird, fühlt die Hand, und zugleich sieht man die Pupille des entsprechenden Auges sich schnell erweitern. Ist die Durchtrennung nicht vollständig, so wird die Pupille nach einiger Zeit unter den Lichteinflusse wieder bedeutend enger. In solchem Falle muss die Durchschneidung wiederholt werden. Die Operation ist von keiner besonderen Blutung: begleitet; das Gehirn wird ebenfalls unbedeutend gequetscht, und andere Nerven werden gar nicht lädirt. Die anatomischen Verhältnisse in dieser Gegend des Sinus cavernosus sind so bequem, dass die Operation leicht von Statten geht, wenn dem Operateur es nur gelingt, den dritten Trigeminusast zu erblicken.

Nach der Durchschneidung des Oculomotorius gab die Sympathicusreizung, bei verdecktem gleichseitigem Auge, keine Verengerung der contralateralen Pupille. War das gleichseitige Auge unbedeckt, so rief die Sympathicusreizung stets bei ihr Erweiterung und Verengerung der contralateralen Pupille hervor. Dagegen blieb 
die Sympathicusreizung an der entgegengesetzten Seite des durchschnittenen Oculomotorius, sowohl bei verdecktem wie offenem Auge der gereizten Seite, auf das Auge mit dem durchschnittenen Oculomotorius immer resultatlos. Gewöhnlich fand an einem Thiere ein Versuch statt, doch habe ich einen Versuch gegen den Schluss der Untersuchung mit verschiedenen Variationen an einem und demselben Thiere ausgeführt und will das Protokoll hier vorführen.

Versuch 37. 22. Februar 1897. Eine 2400 g schwere Katze. Vorbereitungen zum Versuch wie früher (vgl. Versuch 5). 2 cc $8 \%$ ige Curare-Lösung in die Vene; künstliche Athmung. Isolation des obersten Halsknotens. Der obere Abschnitt des Vagus ganz entfernt. Oberhalb des Sympathicusknotens, zu seinen beiden Seiten, werden zwei tiefe, bis auf die Knochen reichende Finschnitte mit der Scheere gemacht. Das linke Auge wie im 5. Versuch geschlossen.

\begin{tabular}{|c|c|c|c|c|}
\hline Zeit & $\begin{array}{c}\text { Gang } \\
\text { der Untersuchung }\end{array}$ & 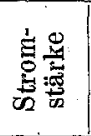 & 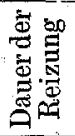 & Beobachtungsresultate \\
\hline $\begin{array}{c}\text { Von } \\
1 \mathrm{~h} 24^{\prime}-1 \mathrm{~h} 25^{\prime}\end{array}$ & & & & $\begin{array}{l}\text { Der Durchmesser der } \\
\text { rechten Pupille } 2 \mathrm{~mm} \text {. }\end{array}$ \\
\hline $1 \mathrm{~h} 25^{\prime}$ & $\begin{array}{l}\text { Reizung des linken } \\
\text { Sympathicus. }\end{array}$ & 15 & 30 & $\begin{array}{l}\text { Die rechte Pupille ver- } \\
\text { engte sich von } 2 \mathrm{~mm} \text { bis } \\
\text { auf } 11 / 3 \mathrm{~mm} \text {. }\end{array}$ \\
\hline $2 \mathrm{~h}$ & $\begin{array}{l}\text { Der linke Oculomo- } \\
\text { torius bei seinem Eintritt } \\
\text { in den Sinus cavernosus } \\
\text { von der Seite her durch- } \\
\text { schnitten. }\end{array}$ & & & $\begin{array}{l}\text { Die linke Pupille erwei- } \\
\text { terte sich; weder directe } \\
\text { noch consensuelle Licht- } \\
\text { reaction. Die Erweiterung } \\
\text { hielt den ganzen Versuch }\end{array}$ \\
\hline $2 \mathrm{~h} 8^{\prime}$ & $\begin{array}{l}\text { Das linke Auge ge- } \\
\text { schlossen. }\end{array}$ & & & 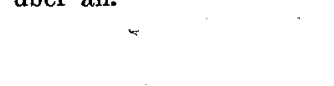 \\
\hline $2 \mathrm{~h} 10^{\prime}$ & $\begin{array}{l}\text { Reizung des linken } \\
\text { Sympathicus. }\end{array}$ & 12 & 60 & $\begin{array}{l}\text { Die rechte Pupille er- } \\
\text { weiterte sich von } 2^{1 / 3} \mathrm{~mm} \\
\text { bis auf } 3 \mathrm{~mm} \text { and ver- } \\
\text { engerte sich darauf bis } \\
\text { auf } 11 / \mathrm{sm} \text {. }\end{array}$ \\
\hline $2 \mathrm{~h} 25^{\prime}$ & $\begin{array}{l}\text { Reizung des linken } \\
\text { Sympathicus. }\end{array}$ & $\begin{array}{c}\text { Von } \\
12--8\end{array}$ & 60 & $\begin{array}{l}\text { Die rechte Pupille ver- } \\
\text { engerte sich von } 6^{1 / 2 ~ m m} \\
\text { bis auf } 51 / 2 \mathrm{~mm} \text { und her- } \\
\text { nach bis auf } 5 \mathrm{~mm} \text { und } \\
\text { blieb so } 5 \text { Minuten lang. }\end{array}$ \\
\hline $2 \mathrm{~h} 31^{\prime}$ & $\begin{array}{l}\text { Der rechte Sympathicus } \\
\text { vorbereitet. }\end{array}$ & & & $\therefore$ \\
\hline
\end{tabular}


Beiträge zur Erforschung des Sympathicuseinflusses auf die contral. Pupille. 241

\begin{tabular}{|c|c|c|c|c|}
\hline Zeit & $\begin{array}{c}\text { Gang } \\
\text { der Untersuchung }\end{array}$ & 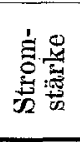 & 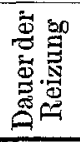 & Beobachtungsresultate \\
\hline $2 \mathrm{~h} 50^{\prime}$ & Beide Augen offen. & & & $\begin{array}{l}\text { Sowohl die directe wie } \\
\text { die consensuelle Reaction } \\
\text { träge. }\end{array}$ \\
\hline $2 \mathrm{~h} 52^{\prime}$ & $\begin{array}{l}\text { Reizung des rechten } \\
\text { Sympathicus. }\end{array}$ & 15 & 10 & $\begin{array}{l}\text { Die linke Pupille be- } \\
\text { wegungslos, } 9 \mathrm{~mm} \mathrm{im} \\
\text { Durchmesser. } \\
\text { Die rechte Pupille wurde } \\
\text { etwas weiter. }\end{array}$ \\
\hline $2 \mathrm{~h} 54^{\prime}$ & 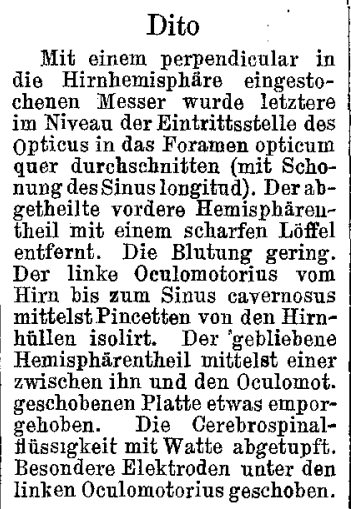 & 8 & 10 & 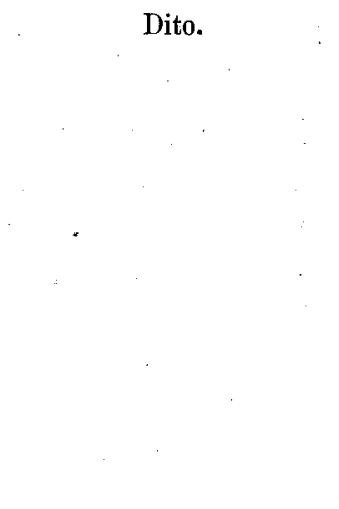 \\
\hline 3 h $10^{\prime}$ & $\begin{array}{l}\text { Reizung des N. oculo- } \\
\text { motorius sinist. }\end{array}$ & 15 & 10 & $\begin{array}{l}\text { Die rechte Pupille ver- } \\
\text { engerte sich langsam ein } \\
\text { wenig. }\end{array}$ \\
\hline 3 h $12^{\prime}$ & Dito & 15 & 10 & Dito \\
\hline $3 \mathrm{~h} 14^{\prime}$ & Dito & 8 & 8 & $\begin{array}{l}\text { Starke und schnelle Ver- } \\
\text { engerung der rechten } \mathrm{Pu} \text { - } \\
\text { pille. }\end{array}$ \\
\hline $3 \mathrm{~h} 15^{\prime}$ & Dito & 7 & 10 & Dito \\
\hline
\end{tabular}

Die Operation wurde durch sorgfältiges Präpariren in Essigsäure-Lösung controlirt.

Auf Grund dieses und ähnlicher Versuche glaube ich annehmen zu dürfen: 1) dass im Oculomotorius das Vorhandensein sympathischer Fasern, welche sich ihm im Sinus cavernosus hinzugesellen und zu seinen Kernen verlaufen, zugelassen werden muss; 2) dass der Sympathicus seinen Finfluss auf das contralaterale Auge bauptsächlich durch diese im Oculomotorius zu dessen Centren verlaufenden Fäden ausübt. 
Hieraus ersieht man ferner, dass die Durchschneidung des contralateralen Oculomotorius die in Rede stehende Verengerung ausbleiben lässt; folglich kann* letztere nicht durch die Herabsetzung des Tonus des Erweiterungscentrums und ebenso nicht durch die im ersten Trigeminusaste zum Auge verlaufenden Verengerer ( $\mathrm{H} \mathrm{all}^{62}$ ), Budge ${ }^{34}$ ), Gruenhagen ${ }^{55}$ ), Eckhard ${ }^{63}$ )) zu Stande kommen, sondern ist das Resultat des Einflusses der im Oculomotorius verlaufenden Verengerer. Das stimmt auch mit den Beobachtungen von Joh. Dogiel überein, welcher bemerkt hat, dass die Erweiterung der Pupille durch Atropin (Paralyse des Oculomotorius) das Auftreten der in Rede stebenden Verengerung verhindert. Die Existenz der sympathischen Fasern im centralen Oculomotoriusstumpfe (bis zum Sinus cavernosus) könnte man nach der Marchi'schen Methode nachweisen. Doch die Untersuchung entsprechender Präparate lehrt, dass in diesem Oculomotoriusabschnitte schon bei normalen Thieren eine gewisse Menge von Markschollen vorhanden ist. Nach der Enucleation des einen Auges ist die Menge der Markschollen in der Oculomotoriuswurzel der anderen Seite fast ebenso gross. Man könnte noch den obersten Halsknoten entfernen und die Präparate hernach nach der Marchi'schen Methode untersuchen, welche Versuche ich in der nächsten Zukunft vorzunehmen gedenke.

Aus der Literatur wissen wir ferner, dass in den peripheren Oculomotoriusabschnitten, besonders an seinen Theilungsstellen, Nervenzellen vorkommen, was ebenfalls gewissermaassen für den Gehalt dieses Nerven an sympathisehen Fasern spricht. Ich habe aber in dem Abschnitte des Oculomotorius, welcher zwischen dem Sinus cavernosus und dem Gehirne liegt, keine Nervenzellen auffinden können. Die Präparate waren mit $1 / 2 \%$ Osmiumsäure-Lösung gefärbt und wurden nach entsprechender Bearbeitung in Paraffin eingeschlossen und mittelst eines Mikrotoms zerschnitten.

Geht also die Reizung des Sympathicus auf das andere Auge durch jene im Oculomotoriusstamme zu dessen Kernen verlaufenden sympathischen Fasern, so entsteht die Frage, wie diese Reizung auf den anderen Oculomotorius übermittelt wird.

Eine solche Uebermittelung kann entweder durch die Commissuren zwischen den beiderseitigen Oeulomotoriuskernen oder dadurch, dass ein Theil des einen Oculomotorius zu den Kernen des anderen geht, zu Stande kommen. Nussbaum ${ }^{64}$ ) studirte im 
Oberstein er'schen Laboratorium das Verhältniss der Wurzelfasern zu den Kernen des Oculomotorius an 8- und 18tägigen Katzen und fand in der Kerngegend des Oculomotorius Kreuzung dünner Fasern. "Diese Fasern entstehen in den Kernen jeder Seite, ziehen zur Raphe, durch welche sie zum Kerne der anderen Seite gehen." $\mathrm{Nussbaum}$ ist es jedoch nicht gelungen, eine einzelne Faser an der ganzen Strecke zu verfolgen. Fr nimmt jedoch Commissurfasern zwischen den Oculomotoriuskernen an. $O$ berste in e ${ }^{20}$ ) hält diese Untersuchungen nicht für beweisend genug und verlangt, dass sie noch eine Bestätigung erfahren sollen. Ich habe an mit Methylenblau bearbeiteten Katzengehirnen wirkliche Commissuren nicht zu sehen bekommen, obwohl ich über 20 Thiere untersucht habe. Man sieht wohl zuweilen eine Nervenfaser, welche von einer Zelle des Ocumolotoriuskernes entspringt und horizontal verläuft, aber ihrè Endigung in den Zellen des contralateralen Oculomotoriuskernes war nicht zu constatiren. An Schnitten aus nach Marchi bearbeiteten Präparaten trifft man oft myelinhaltige Nervenfasern, welche durchweg dunkel gefärbt erscheinen und von rechts nach links verlaufen; gelingt es aber diese Faser weiter zu verfolgen, so sieht man, wie sie mit oder ohne Ueberschreitung der Raphe sich zum Austritt aus dem Hirn umbiegt, somit eine einfache Wurzelfaser darstellt.

Stellen wir also auf Grund eigener Untersuchungen die Existenz der Commissurfasern zwischen den Oculomotoriuskernen in Abrede, so bleibt uns nur noch die Annahme der Kreuzung der Wurzelfasenn des Oculomotorius übrig, um die Uebermittelung der Reizung von einem Oculomotorius auf den andern zu erklären.

In der That hat $\mathrm{Gudden}{ }^{65}$ ) schon bei Kaninchen gefunden, dass jeder Oculomotorius aus einem ventralen Kern seiner und einem dorsalen der contralateralen Seite entspringt. "Diese partielle Kreuzung der Oculomotoriusanfänge ist von allen späteren Untersuchern an verschiedenen Thieren unzweifelhaft demonstrirt worden." Meine früher erwähnten Präparate von Kaninchen haben mich ebenfalls davon überzeugt, dass bei diesen Thieren eine partielle Kreuzung der Wurzelfasern des Oculomotorius besteht. Die die Mittellinie überschreitenden Fasern werden jedoch nur in den hinteren $\mathrm{Ab}$ schnitten der Kerne angetroffen, wie das übrigens fast alle Forscher gefunden haben (Gudden, Perlia etc.). Die pupillenverengernden Fasern scheinen in den vorderen Kernabschnitten des Oculomotorius vorzukommen; wenigstens sprechen hierfür die Versuche von 
Christiani ${ }^{39}$ ), Hensen und Völckers ${ }^{22}$ ) an Hunden und die Beobachtungen von Kahler und $\mathrm{Pick}^{66}$ ) an Menschen. Der Zusammenhang der gekreuzten, in einem Oculomotorius zum Centrum hin verlaufenden Fasern mit den Kernen der Pupillenfasern des anderen muss somit vermittelst der längsverlaufenden, hier in grosser Menge vorkommender Fasern zu Stande kommen. Diese Fasern werden zu den die eine Gruppe der Kerne mit der anderen verbindenden Elementen gezählt (Oberstein e $\mathbf{r}^{20}$ ).

Es erscheint mir, dass nur hierdurch die Resultate solcher Versuche wie desjenigen, in welchem die Reizung des centralen Oculomotoriusstumpfes an seiner Eintrittsstelle in den Sinus cavernosus, bei $15 \mathrm{~cm}$ Spiralenabstand, Verengerung der contralateralen Pupille gibt, erklärlich werden. Selbstverständlich muss bei solchen Versuchen, wegen der geringen Länge des gereizten Nerven und der Nähe des Gehirns, besondere Aufmerksamkeit einer sorgfältigen Isolation der Leiter und des gereizten Nerven geschenkt werden. Aus diesem Grunde habe ich zu diesem Versuch besondere Elektroden construirt: an ein Glasrohr wurde eine dünne, rinnenförmige, ca. $2 \mathrm{~mm}$ breite Glasplatte angelöthet. Das Ende der Rinne war nach oben gebogen; in der Rinne befanden sich die Elektroden. Nach der Befreiung des Oculomotorius von den Gehirnhäuten wurde diese Glasrinne derart zwischen den Nerven und das Gehirn geschoben, dass die Elektroden vom Gehirn durch die Glasrinne getrennt waren.

Schliesslich möchte ich noch auf ein Moment hinweisen, welches vielleicht ebenfalls einen verengernden Einfluss auf die Pupille ausüben könnte: ich meine eben die Wirkung der pupillenerweiternden Centren der Hirnrinde auf den Tonus der Oculomotoriuscentren. Es wäre nämlich möglich, dass unter dem Einfluss der Sympathicusreizung der Tonus der Rindencentren herabgesetzt würde, wodurch gewissermaassen ihr hemmender Einfluss auf die Oculomotoriuscentren paralysirt werden könnte.

Die Controle dieser für sich nicht unwahrscheinlichen Voraussetzung erfordert selbstverständlich eine Reihe von Versuchen, welche ich aus Mangel an Zeit und in Anbetracht dessen, dass von mir schon einige positive Resultate in dieser Beziehung erzielt worden sind, nicht vornehmen konnte.

Die im physiologischen Abschnitt meiner Abhandlung vorgeführten Versuche erlauben mir, zu behaupten, dass die schon 1893 
Beiträge zur Erforschung des Sympathicuseinflusses auf die contral. Pupille. 245

von Joh. Dogiel angegebene Pupillenverengerung in Folge der Sympathicusreizung an der contralateralen Seite

1) nicht durch die Veränderungen in dem an der Seite des gereizten Sympathicus befindlichen Auge bedingt ist,

2) nicht ganz als eine Folge der consensuellen Pupillenreaction, wie Schenk und Fuss es annehmen, aufgefasst werden kann,

3) wahrscheinlich nicht von den Veränderungen in dem zu dem gereizten Sympathicus contralateralen Auge abhängt,

4) eine neu entdeckte, durch die in der Gegend des Sinus cavernosus den Hirnnerven sich hinzugesellenden Sympathicusfasern vermittelte Reflexerscheinung darstellt,

5) allem Anschein nach hauptsächlich durch jene (von mir vorausgesetzten) Sympathicusfasern, welche im Sinus cavernosus zum Oculomotorius sich hinzugesellen und in seinem Stamm zu dessen Centren verlaufen, zu Stande kommt,

6) unter dem Einfluss der pupillenverengernden Oculomotoriusfasern zu Stande kommt.

Ausserdem erwies es sich,

7) dass die Hauptmasse der Sympathicusfasern (in der Form eines ganzen Bündels) aus dem oberen Halsknoten (bei Katzen) nicht mit der Art. carot. int., sondern auf einem ganz anderen, ähnlich dem von Fr. Franck angegebenen Wege in den Sinus cavernosus gelangen, und

8) dass die Pupillenerweiterer sogleich vor dem Ganglion Gasseri in der Form einiger Bündel oder Fasern verlaufen.

\section{Erklärnng der Abbildungen.}

Fig. 1. Verlauf des Tract. peduncul. transv. Hartnack Oc. 3 Syst. 2 (die Zeichnung hernach verkleinert). $c q a=$ vorderer Zweihügel; $b r c a=$ vorderer Bindearm; $p c=$ pedunculus conarii; $G=$ Ganglion, aus welchem ein Theil des Tract. peduncul. transv. seinen Anfang nimmt; $\operatorname{tr} p t=$ Tract. peduncul. transv.

Fig. 2. Nervenzellen aus dem Ursprungskern des Tract. peduncul. transv. (Fig $1 G$ ); $a b c=$ Nervenzellen mit zur Peripherie gerichteten Achsencylinderfortsätzen; $d=$ Nervenzelle mit zur Mittellinie gerichtetem Achsencylinderfortsatz. Zeiss. Comp. Oc. 4 Homog. Imm. Apochrom. 2,0 mm. 
Fig. $3 a$ und Fig. $3 b$. Nervenzellen von der Oberfläche des vorderen Zweihügels. Man erblickt das pericelluläre Netz. Zeiss. Comp. Oc. 12 Homog. Imm. Apochrom. 2,0 mm.

Fig. 4. Endpinselchen aus dem vorderen Zweihügel der Katze. Zeiss. Comp. Oc. 4 Apochrom. $2,0 \mathrm{~mm}$.

Fig. $5 a b c d$. Endigungen der Fasern des Tract. opt. im äusseren Kniehöcker; $d=$ Endigung eines dünnen, von der Schlängelung beginnenden Fadens; e $f=$ Endigung der aus den medialen Hirntheilen stammenden Fasern im äusseren Kniehöcker; bei $f$ sieht man die Bildung einer sich schwach färbenden Faser aus einer gewöhnlichen, myelinhaltigen; $a b$ c e $f=$ Zeiss. Comp. Oc. 4 Homog. Imm. 2,0 mm; $d=$ Zeiss. Comp. Oc. 8 Apochrom. $4 \mathrm{~mm}$.

Fig. 6. Mikrophotographie von der oberen Conariumfläche der Katze; $c p c=$ Commissura peduncul. conarii; $v=$ Gefäss; $n e=$ Nervenfasern, welche an der oberen Conariumfläche Schlingen bilden.

Fig. 7. Nervenzellen ans dem Conarium. An den Zellen $c$ und $d$ sieht man die sie umflechtenden pericellulären Fäden. Bei der Zelle $d$ bemerkt man die Endverdickung des pericellulären Fadens. Zeiss. Comp. Oc. 4 Homog. Imm. $2,0 \mathrm{~mm}$.

Fig. 8. Freie Nervenendigung auf der oberen Fläche des Conariums. Zeiss. Comp. Oc. 4 Homog. Imm. 2,0 mm.

Fig. 9a. Nervenzelle von der unteren Conariumfläche; ihr Achsencylinderfortsatz kann bis zu seiner Endverzweigung verfolgt werden; $b=$ Endigung der Nervenfaser in der Nähe der Nervenzelle $a ; c=$ Endigung, welche wahrscheinlich ebenfalls einem Achsencylinderfortsatz einer Nervenzelle des Conariums angehört. Zeiss. Comp. Oc. 4 Homog. Imm. $2,0 \mathrm{~mm}$.

Fig. 10. Verlauf des Sympathicus bei der Katze oberhalb des Gangl. cervic. super. Gezeichnet mittelts der Lupe. $I I I=\mathrm{N}$. oculomotorius; $I V=\mathrm{N}$. trochlearis; $V I=$ N. abducens; $g G=$ Gangl. Gasseri; $b o=$ bulla ossea; $g s=$ gangl. cervic. super. n. sympath.; $g v=$ plexus ganglioformis n. vagi; $a c=$ art. carotis commonis; $m a i=$ meatus acust. int. ; ot $t=$ abgekniffene hintere Fläche des Schläfenbeines; $n=$ Zweige des gangl. cervi. super.: einer zum Wirbelkanal, der andere zu einem der Cervicalnerven; $n s=$ Nerven, welche vom oberen Ende des oberen Halsknotens beginnen und längs der bulla ossea verlaufen; $n^{\prime} s^{\prime}=$ Verlauf derselben Nerven unterhalb des Gangl. Gasseri im Sinus cavernosus, zu sehen erst, nachdem das Gangl. Gasseri nach aussen gewendet ist; $n a=$ Nervenzweige vom Gangl. cervic. super. zu den Gefässen; $s=$ ein Theil des Halssympathicus.

Fig. 11. Verlauf der Sympathicusfäden im linken Sinus cavernosus; das Gangl. Gasseri ist nach aussen gedreht. Gezeichnet unter der Lupe. Die Buchstaben wie in Fig. 10. 
Beiträge zur Erforschung des Sympathicuseinflusses auf die contral. Pupille. 247

\section{Literaturverzeichniss.}

1) Zeitschr. f. Psychiatrie Bd. 42. S. 480. Neurolog. Centralblatt 1885 Nr. 19.

2) Versamml. d. deutsch. Naturforsch. u. Aerzte in Strassburg vom 18.-25. Sept. 1885.

3) Arch. f. Psychiatrie u. Nervenkrankh. Bd. 20 s. 714.1889.

4) Arch. f. d. ges. Physiologie Bd. 31 S. 60. 1883.

5) Bechterew, Die Leitungsbahnen im Gehirn u. Rückenmark. Șt. Petersburg 1896.

6) Centralbl. f. d. med. Wissensch. 1878 Nr. 25 u. Arch. de Physiol. norm. et path. S. 658 . 1878 .

7) Klinische Zeitung 1883, Januar (russ.).

8) Neurolog. Centralb. S. 802-809. 1894.

9) Sitzungsprotokolle d. Gesellsch. d. Psychiater in St. Petersburg S. 43 (russ.) 1883.

10) Pribytkow, Ueber den Sehnervenfaserverlauf und über ihren Endigungsort in den subcorticalen Centren Diss. (russ.). Moskau 1895.

11) Longet, Anat. u. Physiologie des Nervensystems, übersetzt von Hein, Bd. 2. 1849.

12) Budge, Ueber die Bewegung der Iris. 1855.

13) Beiträge zur Anat. u. Physiologie von C. Eckhardt Bd. 4. S. 109.1869.

14) Eckhardt, Beiträge zur Anatomie und Physiologie. Giessen 1885.

15) Darkschewitsch, Ueber die Leiter der Lichtreizung von der Netzhaut auf der Oculomotorius (russ.). Moskau 1887.

16) Archiv: f. d. ges. Physiologie Bd. 47 S. 289.1890.

17) Henschen, Klin. u. anat. Beiträge zur Path. d. Gehirns. Upsala. I. Theil 1890, II. Theil 1892 u. III. Theil 1894.

18) Flourens, Rech. experim. sur 1 , propr. et 1 . fonct. de syst. nerveux. Paris 1892.

19) Meynert, Stricker's Gewebelehre Bd. 2 S. 732.

20) Obersteiner, Handb. d. Lehre v. Bau d. centr. Nervensystems S. 390.1896.

21) Koelliker, Handb. d. Gewebelehre d. Menschen Bd. 2. 1696.

22) Graefe's Arch. Bd. 24 S. 1-27.

23) Wratschr. $1883 \mathrm{Nr} .35$ (russ.).

24) Stilling, Untersuch. üb. d. Bau d. opt. Centralorg. I. Theil S. 15. Berlin 1892.

25) Neurolog. CentralbI. 1887 Nr. 20.

26) Neurolog. Centralbl. 1888 Nr. 22.

27) Edinger, Vorl. üb. d. Bau d. Nervencentren. 1893.

28) Deutsche med. Wochenschr. $1889 \mathrm{Nr} .47$.

29) Arch. f. Ophth. Bd. 35 Ab. 4. 1889.

30) Arch. f. Psychiatrie Bd. 2 S. 364.

31) Gall u. Sporzheim, Anat. et physiol. du syst. nerv. Paris 1810.

32) Arch. f. Psychiatrie u. Nervenkrankh. Bd. 13 S. 341.1882.

33) Arch. f. Psychiatrie Bd. 14 S. 699.1883.

E. Pflūger, Archiv für Physiologie. Bd. 69. 
248 N. Tümianzew: Beitr. z. Erforsch. d. Sympathicuseinfl. a. d. contral. Pupille.

34) Denkschr. d. Naturwiss. Cl. d. k. Akad. d. Wiss. in Wien Bd. 55.

35) Krause, Anat. d. Kaninchens. Leipzig 1884.

36) Arbeit d. physico-medic. Gesellsch. S. 261 (russ.). 1867.

37) Südruss. med. Zeitung 1892 Nr. 3 (russ.).

38) Neurolog. Centralbl. S. 555. 1886.

39) Arch. f. Psychiatrie H. 2. 1896.

40) Neurolog. Centralbl. S. 820. 1893.

41) Pedro Ramòn, Inv. s. v. centros opticos de los vertebrados. Diss. 1890.

42) S. Ramòn y Cajal, Beitrag zum Studium der Med. obl., des Kleinhirns u. d. Ursprungs der Gehirnnerven. Leipzig 1896.

43) Neurolog. Centralbl. 1896 Nr. 19 u. Arch. f. Psychiatrie Bd. 28 H. 1.

44) Medicin. Uebersicht, Januar 1897 (russ.).

45) Centralblatt f. prakt. Augenheilkunde S. 19. 1894.

46) Arch. f. mikr. Anat. Bd. 47. S. 707. 1896.

47) Arch. f. Psychiatrie Bd. 27 H. 1 u. 2. 1895.

48) Gazeta Lekarska 1893 Nr. 43 und Neurolog. Bot. Bd. 2 L. 2 (russ). 1894.

49) Arch. f. d. ges. Physiologie Bd. 56. 1894.

50) Arch. f. d. ges. Physiol. Bd. 62 S. 494.1895.

51) Gazeta Lekarska 1986.

52) Filin sson. Ueber die vasomotorischen Nerven der Retina. Kasan. S. 8 Diss. (russ.) 1896.

53) Bellärminow. Versuch zu der Anwendung der graphischen Methode bei der Untersuchung der Pupille und des intraocularen Druckes (verm. d. Photographie). St. Petersburg 1886. Diss. (russ.).

54) Leblanc. Essai sur l. modific. de la pupille . . Paris 1875.

55) Braunstein. Zur Lehre von der Innervation der Irisbewegungen. Charkow 1893 (russ).

56) Pflüger's Archiv Bd. 52 S. 555.

57) Centralbl. f. d. med. Wiss. Nr. 37 S. 577. 1863.

58) Travaux du laboratoire de M. Marey. IV. Paris 1880.

59) Arch. f. d. ges. Physiologie Bd. 47 S. 108.

60) A damük. Sep.-Abdr. aus d. 59. Bde. d. Sitzungsber. d. k. Akad. d. Wiss. Abth. II. Februarheft 1869.

61) Beilage zum 66. Bde. d. Schriften d. kais. Akad. d. Wiss. zu St. Petersburg. 1891.

62) Eckhardt's Beiträge zur Anat. u. Physiol. Bd. 12 S. 135.

63) Centralbl. f. Physiol. 1892 Juni S. 129.

64) Medicin. Jahrb. 1887 S. 487.

65) Tagbl. d. Vers. d. Naturf. in Salzburg 1881 S. 186.

66) Arch. f. Psychiatrie Bd. 10 S. 179 und 297. 1880. 